\title{
RESEARCH
}

Open Access

\section{Lentivirus-mediated downregulation of a-synuclein reduces neuroinflammation and promotes functional recovery in rats with spinal cord injury}

Hong Zeng, Nan Liu, Yan-yan Yang, Hua-yi Xing, Xiao-xie Liu, Fang Li, Gao-yan La, Meng-jie Huang and Mou-wang Zhou* (i)

\begin{abstract}
Background: The prognosis of spinal cord injury $(\mathrm{SCl})$ is closely related to secondary injury, which is dominated by neuroinflammation. There is evidence that a-synuclein aggregates after $\mathrm{SCl}$ and that inhibition of a-synuclein aggregation can improve the survival of neurons after $\mathrm{SCl}$, but the mechanism is still unclear. This study was designed to investigate the effects of a-synuclein on neuroinflammation after $\mathrm{SCl}$ and to determine the underlying mechanisms.

Method: A T3 spinal cord contusion model was established in adult male Sprague-Dawley rats. An SNCA-shRNAcarrying lentivirus (LV-SNCA-shRNA) was injected into the injury site to block the expression of a-synuclein (forming the $\mathrm{SCl}+\mathrm{KD}$ group), and the $\mathrm{SCl}$ and sham groups were injected with an empty vector. Basso-Beattie-Bresnahan (BBB) behavioural scores and footprint analysis were used to detect motor function. Inflammatory infiltration and myelin loss were measured in the spinal cord tissues of each group by haematoxylin-eosin (HE) and Luxol Fast Blue (LFB) staining, respectively. Immunohistochemistry, Western blot analysis, and RT-qPCR were used to analyse protein expression and transcription levels in the tissues. Immunofluorescence was used to determine the morphology and function of glial cells and the expression of matrix metalloproteinase-9 in the central canal of the spinal cord. Finally, peripheral serum cytokine levels were determined by enzyme-linked immunosorbent assay.

Results: Compared with the SCI group, the SCl+KD group exhibited reduced inflammatory infiltration, preserved myelin, and functional recovery. Specifically, the early arrest of a-synuclein inhibited the pro-inflammatory factors IL$1 \beta$, TNF- $a$, and IL-2 and increased the expression of the anti-inflammatory factors IL-10, TGF- $\beta$, and IL-4. The neuroinflammatory response was regulated by reduced proliferation of Iba $1+$ microglia/macrophages and promotion of the shift of M1-polarized Iba1+/iNOS+ microglia/macrophages to M2-polarized Iba1+/Arg1+ microglia/macrophages after injury. In addition, compared with the SCl group, the SCl+KD group also exhibited a smaller microglia/astrocyte (Iba1/GFAP) immunostaining area in the central canal, lower MMP-9 expression, and improved cerebrospinal barrier function.

Conclusion: Lentivirus-mediated downregulation of a-synuclein reduces neuroinflammation, improves bloodcerebrospinal barrier function, promotes functional recovery, reduces microglial activation, and promotes the polarization of M1 microglia/macrophages to an M2 phenotype to confer a neuroprotective immune microenvironment in rats with $\mathrm{SCl}$.
\end{abstract}

Keywords: Spinal cord injury, a-Synuclein, Microglia, Astrocyte, Blood-cerebrospinal barrier, Matrix metalloproteinase

\footnotetext{
* Correspondence: zhoumouwang@163.com

Department of Rehabilitation Medicine, Peking University Third Hospital, 49 North Garden Road, Beijing 100191, China
}

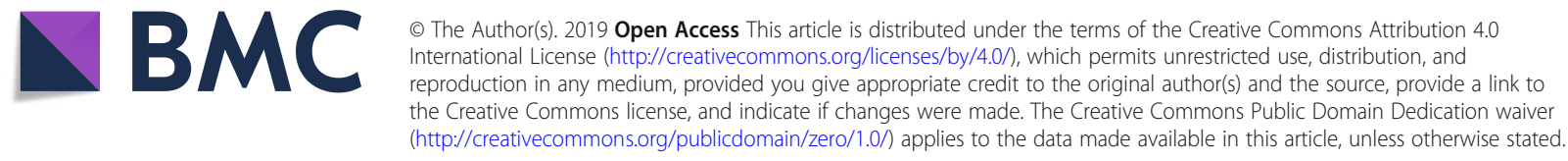




\section{Introduction}

Traumatic spinal cord injury (SCI) is caused by an external force applied directly or indirectly to the spinal cord. Neuronal loss, axonal degeneration, and demyelination at the injury site cause functional or organic changes in the spinal cord. Although modern medical rehabilitation has improved patient outcomes and prognosis, drug therapies to prevent neuronal death or promote regeneration remain limited $[1,2]$. Studies have shown that in the context of SCI, mechanical damage causes neurons and glia at the injury site to die within minutes to hours. This death is followed by a delayed secondary injury phase in which the characteristic neuroinflammatory response persists. SCI-induced glial activation and the subsequent release of inflammatory factors such as interleukin (IL), tumour necrosis factor (TNF), and interferon (IFN) accelerate neuronal death while inducing vascular endothelial cells to express multiple cell adhesion and chemotaxis molecules to attract more inflammatory factors. These factors also typically stimulate nitric oxide (NO) release, increase capillary permeability, and cause blood-cerebrospinal barrier (BCSB) dysfunction [3, 4]. SCI triggers a range of cellular and molecular events, including microglial/astrocyte activation, peripheral bloodderived macrophage infiltration, pro-inflammatory/antiinflammatory response imbalance, abnormal mitochondrial activity, oxidative stress, abnormal protein aggregation, and free radical toxicity. These processes induce neuronal death and lead to permanent neurological deficits. The amplification of the inflammatory response is the main cause of secondary injury, and the secondary injury process worsens outcomes. Therefore, improving the immune environment of the spinal cord during secondary injury is the most urgent and important goal of treatment [5].

Neuroinflammation originates from innate immunity of the central nervous system (CNS). Microglia are the most common immune cells in the CNS. Under normal conditions, microglia are in a quiescent state; however, after injury or infection, microglia are activated into effector cells with immune functions. At present, two forms of microglial/macrophage polarization are recognized: (1) "classic" M1 polarization, which occurs through the pro-inflammatory pathway and produces inflammatory cytokines, reactive oxygen species, and free radicals, and (2) "alternative" M2 polarization, which occurs through the anti-inflammatory pathway and is often associated with the anti-inflammatory response, tissue repair, and metabolic homeostasis [6]. An increasing number of animal experiments have found that cytokines can be modulated to alter the expression of different microglial subtypes, thereby activating different polarization pathways after nerve injury. In addition, many studies have found that reactive astrocytes in SCI models participate in the development of neuroinflammation in the acute and subacute phases; the chronic phase is closely related to scar formation after injury and hinders nerve regeneration and repair [7]. Furthermore, secondary damage involves not only upregulation of pro-inflammatory factors but also cell excitotoxicity, metabolic failure, and impairment of the clearance of protein deposits after cell death, all of which cause acute and chronic neuroinflammation [7].

$\alpha$-Synuclein $(\alpha-S y n)$ is a soluble protein expressed in the presynaptic and perinuclear phases of nerves. Its accumulation has been associated with many degenerative neuropathies. The structure of $\alpha$-Syn depends largely on the intracellular environment in which it is located, and the protein exhibits different structural forms, such as monomers, oligomers, fibrils, and fibres. Synaptic nucleoproteins aggregate easily under pathological conditions. The formation of an insoluble fibrin precipitate eventually leads to the death of nerve cells, and the aggregation of $\alpha$-Syn increases mitochondrial dysfunction, oxidative stress, apoptotic pathway activation, and glial activation in the CNS [8]. SNCA, a key gene that encodes the $\alpha$ Syn protein, can be inhibited by lentivirus-mediated SNCA short hairpin RNA (LV-SNCA-shRNA) [9]. Within $\alpha$-Syn, the Ser-129 residue can be phosphorylated by G-coupled receptor protein kinases (GCRPs), and this phosphorylation promotes the oligomerization of $\alpha$-Syn fibre filaments by altering the C-terminal potential distribution and hydrophobicity of $\alpha$-Syn [10]. $\alpha$ Syn that is phosphorylated at the Ser-129 site is widely and selectively distributed in many synaptic pathological lesions, such as Lewy bodies [10]. Studies have shown that after SCI, $\alpha$-Syn, knockdown of which inhibits neuronal apoptosis and promotes spontaneous repair, forms punctate aggregates in spinal cord neurons [11]. Another report has shown that $\alpha$-Syn triggers the migration of microglia after SCI [12]. However, the effect of $\alpha$-Syn on neuroinflammation after SCI and its mechanism remains unclear.

Therefore, the goal of this study was to observe $\alpha$-Syn expression and Ser-129 phosphorylation after SCI in vivo. The effects of $\alpha$-Syn on neuroinflammationrelated factors, including microglial/astrocyte activation, phenotype polarization, and MMP-9 expression, were detected following SCI. Furthermore, we explored the effects of $\alpha$-Syn on the BCSB after injury. This study clarifies whether neuroimmunity can be improved by knocking down $\alpha$-Syn.

\section{Materials and methods \\ Animals}

A total of 120 specific pathogen-free (SPF) adult male Sprague-Dawley rats (Department of Animal Science, Peking University School of Medicine, Beijing, China) were used in this study. All rats were housed in separate 
cages under a 12-h light-dark cycle at $23 \pm 1{ }^{\circ} \mathrm{C}$ and $50 \%$ relative humidity, and food and water were available ad libitum. All rats were acclimatized to the environment for at least 1 week prior to the experiment and were maintained as directed by the experimental animal care and use guidelines. The study was approved by the Animal Welfare Ethics Branch of the Peking University Bioethics Committee.

The animals were randomized into the following three groups ( $n=40$ in each group): (1) the sham+LV-pLentU6-Puro group (the sham group); (2) the SCI+LVpLent-U6-Puro group (the SCI group); and (3) the $\mathrm{SCI}+\mathrm{LV}$-SNCA-shRNA group (the $\mathrm{SCI}+$ knockdown [KD] group) (Fig. 1).

\section{Construction of the lentiviral LV-SNCA-shRNA vector} Lentiviruses containing SNCA-shRNA (NM_019169.2) were constructed and synthesized by ShanDong ViGene Co., Ltd. (Shandong, China). The primers for SNCA were as follows: forward, 5'-GTGGCTGCTGCTGAGAAAAC-3' and reverse, 5'-TCCATGAACGACTCCCTCCT-3'. The virus titre of LV-SNCA-shRNA was $1.0 \times 10 \mathrm{E} 9 \mathrm{TU} / \mathrm{ml}$. In addition, an LV-GFP-SNCA-shRNA green fluorescent protein (GFP)-tagged lentivirus was constructed to verify the efficiency of transfection and knockdown.

\section{Surgery and transfection}

All rats received prophylactic antibiotic treatment with ampicillin sodium $(80 \mathrm{mg} / \mathrm{kg}$; Harbin Pharmaceutical
Group Co., Ltd., Harbin, China) for 3 days before SCI surgery. The rats were intraperitoneally injected with $2 \%$ sodium pentobarbital $(0.1 \mathrm{ml} / \mathrm{kg})$ and placed in a prone position on the operating table. The limbs were fixed, and the upper chest was raised with a cotton pad. Along the T2 spine of each rat, the C8-T4 dorsal skin was dissected, the back muscle was peeled off layer by layer, and the T3 segment of the thoracic vertebra was dissected. The spinal cord was removed by performing a laminectomy of the T3 segment under a surgical microscope. In the sham group, the incision was closed layer by layer after the spinal cord was exposed, but no injury was induced. The SCI group was injured with a PSI-IH precision striking device (IH impactor; Precision Systems and Instrumentation, Lexington, KY, USA) after the spinal cord was exposed. The striking head was adjusted over the exposed T3 spinal cord segment, dropped so that it just touched the dural sac, and then raised by 2 $\mathrm{cm}$. The force was set to 400 kilodynes, the compression time was set to $5 \mathrm{~s}$, and the number of hits was set to one. The standards for successful generation of the rat $\mathrm{SCI}$ model were the presence of a contusion at the injury site, convulsions in both lower extremities, and spastic swaying of the tail. The force with which the spinal cords of the rats were impacted was monitored by a computer. Following SCI, $10 \mu \mathrm{l}$ of lentivirus containing the target gene shRNA was injected in situ using a microsyringe. The sham group and the SCI group were given the same dose of an empty lentiviral vector. After
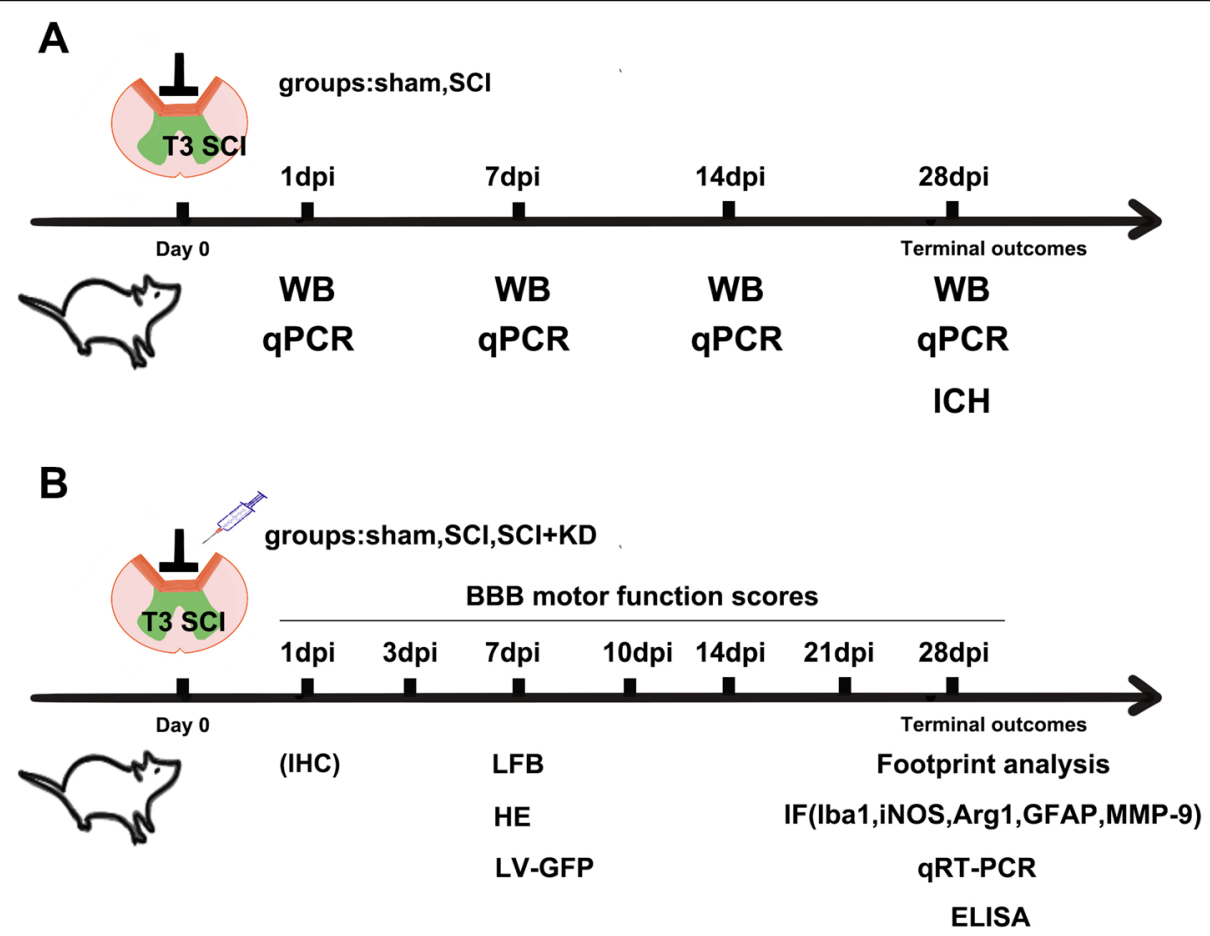

Fig. 1 a, b Timeline of the experimental protocol 
injection, the dorsal tissue of each rat was sutured layer by layer. After the procedure, the rats were allowed to recover on a heated blanket and were placed in a clean cage for observation. Beginning immediately after surgery, the rats were injected subcutaneously with Ringer's sodium lactate solution $(5 \mathrm{ml})$ and ampicillin sodium twice daily (morning and afternoon) until the third day after injury. The bladders of the rats were squeezed 3 times daily after surgery until spontaneous urination was restored. All assessments and analyses were performed by experienced researchers who were unaware of the experimental design.

\section{Behavioural tests}

\section{Hindlimb exercise score}

Basso-Beattie-Bresnahan (BBB) motor function scores were used for evaluation of hindlimb motor function [13]. The rats were placed on a circular platform with a diameter of $2 \mathrm{~m}$. The walking and limb activity scores of the hindlimbs were observed and recorded. In the first stage (0-7 points), the joint activity of the hindlimbs of the animals was scored. In the second stage (8-13 points), the gait and the coordination of the hindlimbs were scored. In the third stage (14-21 points), the fine movements of the claws were judged. The scores for the three stages together totalled 21 points. Each group was scored 1 day before surgery and $1,3,7,10,14,21$, and 28 days post-injury (dpi).

\section{Footprint analysis}

Gait behaviour and motor coordination were assessed on the 28th day after injury [14]. The forelimbs and hind paws were coated with dyes of different colours, and the animals were placed on a $7.5 \mathrm{~cm} \times 100 \mathrm{~cm}$ runway covered with white paper. The animals were encouraged to walk straight to the finish line so that representative images of their gaits could be obtained and coordination could be assessed.

\section{Tissue preparation}

At the predetermined time points, each rat was anaesthetized, the diaphragm was cut, and the pericardium was opened. After blood was collected from the left apex, $5 \mathrm{~mm}$ of the spinal cord above and below the T3 injury site was quickly placed in a cryotube, frozen in liquid nitrogen, and stored in a $-80^{\circ} \mathrm{C}$ low-temperature freezer. In addition, some rats were perfused with 150 $\mathrm{ml}$ of sterile $0.9 \%$ normal saline, and the right atrial appendage was cut at the same time. After the effluent liquid became clear, $300 \mathrm{ml}$ of $4 \%$ paraformaldehyde (Biosharp, Beijing, China) was used for perfusion until the tissues of the rats became hard. The tissue near the injury site at the T3 level ( $5 \mathrm{~mm}$ above and below) was then removed and placed in paraformaldehyde overnight. The tissue was either dehydrated in xylene and gradient alcohol solutions, embedded in paraffin, and cut into $5-\mu \mathrm{m}$ serial sections with a slicer or dehydrated in sucrose solutions $(10 \%, 20 \%$, and $30 \%)$, embedded in optimum cutting temperature (OCT) compound, cut into continuous $20-\mu \mathrm{m}$ frozen sections with a microtome, and processed for immunofluorescence (IF).

\section{Paraffin section histopathological staining}

At $28 \mathrm{dpi}$, paraffin sections from each group were heated at $60^{\circ} \mathrm{C}$, placed in xylene I and II for $30 \mathrm{~min}$, and placed in gradient alcohol solutions of $100 \%, 100 \%, 95 \%, 95 \%$, and $80 \%$ for $5 \mathrm{~min}$ each. The sections were rinsed twice with steamed water.

\section{HE staining}

A series of sections were stained with haematoxylin for $1 \mathrm{~min}$, double-rinsed in distilled water, differentiated in $1 \%$ hydrochloric acid, double-rinsed in distilled water, and stained with eosin (Sigma-Aldrich) for $2 \mathrm{~min}$.

\section{LFB staining}

A series of sections were stained with a $0.1 \%$ Luxol Fast Blue (LFB; Sigma-Aldrich) solution, sealed for $8 \mathrm{~h}$ at $60{ }^{\circ} \mathrm{C}$, washed with distilled water, and placed in $95 \%$ alcohol for $10 \mathrm{~min}$. Each mixture was separated in a $0.05 \%$ lithium carbonate aqueous solution (Leagene, Beijing, China) for $10 \mathrm{~s}$ and in a $70 \%$ alcohol solution for $20 \mathrm{~s}$. The above two steps were repeated until the grey and white matter (GM and WM, respectively) were clearly observable under the microscope.

The above sections were dehydrated by a conventional alcohol gradient $(80 \%, 95 \%, 95 \%, 100 \%, 100 \%$ alcohol for $2 \mathrm{~min}$ ), placed in xylene I and II for $10 \mathrm{~min}$, and then sealed with neutral gum. Images of both haematoxylineosin (HE) staining and LFB staining were captured with a Nano Zoomer Digital Pathology system (Hamamatsu, Japan). Three blinded experimenters used Image-Pro Plus 6.0 (Media Cybernetics, Rockville, MD, USA) to calculate the staining density to quantify the myelin and lesion area. There were five animals per group. For each animal, five spinal cross-sections in the rostral-caudal plane taken from the level of the injury were analysed.

\section{IHC}

For immunohistochemistry (IHC), the $5-\mu \mathrm{m}$ paraffin sections were dewaxed in water, and antigen retrieval was carried out in a pressure cooker with sodium citrate buffer (10 mM, pH 6.0; Boster Biological Technology, Ltd., Wuhan, China). The tissues were then blocked with $3 \% \mathrm{H}_{2} \mathrm{O}_{2}$ to quench endogenous peroxidase activity. The tissues were blocked in 10\% goat serum (Boster 
Biological Technology, Ltd) for $30 \mathrm{~min}$, incubated with a rabbit anti- $\alpha$-Syn antibody (1:2000; Abcam, Cambridge, MA, USA), rabbit anti-Iba1 (1:200; GeneTex, Inc., USA), and mouse anti-glial fibrillary acid protein (GFAP; 1:500; Santa Cruz, Dallas, TX) at $37^{\circ} \mathrm{C}$ for $2 \mathrm{~h}$, rinsed with PBS, and incubated with a goat anti-rabbit IgG secondary antibody (1:100; Zhongshan Jinqiao, Beijing, China) for 30 min. After washing, DAB (Zhongshan Jinqiao) was used for detection. The sections were then rinsed with water, the nuclei were counterstained with haematoxylin, the sections were placed in gradient alcohol solutions for $2 \mathrm{~min}$ each and in xylene I and II for $5 \mathrm{~min}$ each, and the slides were sealed with neutral resin. The sections were examined at $\times 20$ magnification using an electron microscope (ECLIPSE 90i, Nikon, Japan) and Image-Pro Plus 6.0 to detect $\alpha$-Syn staining in the GM and WM (through quantitative analysis of the mean optical density of the $\alpha$-Syn+ cells). There were five animals per group. The average area of 4 different sections from each rat was calculated.

\section{IF}

The frozen sections were thawed at room temperature for $30 \mathrm{~min}$. The sections were washed three times (for $10 \mathrm{~min}$ each) with $0.1 \mathrm{mmol} / \mathrm{l}$ PBS containing $0.1 \%$ Triton X-100 (Sigma-Aldrich) (PBS-TX). The sections were pre-incubated in permeabilization blocking buffer $(0.1$ $\mathrm{mmol} / \mathrm{l} \mathrm{PBS}, \mathrm{pH} 7.3$, containing $0.5 \%$ Triton) for $15 \mathrm{~min}$ at $37^{\circ} \mathrm{C}$. The sections were then blocked with $10 \%(\mathrm{v} / \mathrm{v})$ goat serum (Boster Biological Technology, Ltd) for another $30 \mathrm{~min}$ and incubated overnight with a primary antibody at $4{ }^{\circ} \mathrm{C}$. After washing with PBS-TX the next day (3 times for $10 \mathrm{~min}$ each), the sections were incubated with a secondary antibody for $1 \mathrm{~h}$ at room temperature and then washed with PBS-TX. The nuclei were stained with 4,6-diamidino-2-phenylindole (DAPI, $1 \mu \mathrm{g} / \mathrm{ml}$; Sigma-Aldrich) for $5 \mathrm{~min}$, and the sections were washed with PBS-TX and sealed with an antifluorescence quencher. Images were captured under a Leica DM4 B confocal fluorescence microscope (Leica Microsystems Inc., Wetzlar, Germany) with a Leica TCS SP8 system (Leica Microsystems Inc). Negative controls were incubated with the corresponding isotype serum instead of a primary antibody.

The following primary antibodies were used at the indicated dilutions: mouse anti-ionized calcium-binding adaptor molecule (Iba1; 1:100; Abcam, Cambridge, MA), rabbit anti-Iba1 (1:200; GeneTex, Inc., USA), mouse anti- glial fibrillary acid protein (GFAP; 1:500; Santa Cruz, Dallas, TX), rabbit anti-iNOS (inducible nitric oxide synthase, 1:400; Abcam, Cambridge, MA), rabbit anti-arginase-1 (Arg1; 1:400; GeneTex, Inc., USA), and rabbit anti-matrix metalloproteinase-9 (MMP-9; 1:100; Abcam, Cambridge, MA). The following fluorescent secondary antibodies were used at the indicated dilutions: Alexa Fluor 594-conjugated AffiniPure goat anti-mouse IgG $(\mathrm{H}+\mathrm{L})$ (1:800; Jackson ImmunoResearch Laboratories, West Grove, PA), Alexa Fluor 488-conjugated AffiniPure goat anti-rabbit IgG $(\mathrm{H}+\mathrm{L})$ (1:400; Jackson ImmunoResearch Laboratories), Cy3-conjugated goat anti-rabbit IgG $(\mathrm{H}+\mathrm{L})$ (1:200; Boster Biological Technology, Ltd), and Alexa Fluor 488-conjugated AffiniPure goat anti-mouse IgG $(\mathrm{H}+\mathrm{L})$ (1:400; Jackson ImmunoResearch Laboratories).

There were 5 animals per group, and three sections from each animal at the same level (the injury level) were used to determine the number of positive cells or the mean optical density (mean optical density = integrated optical density (IOD)/area) for fluorescence quantification. The number of Iba1+ cells in each transverse section of the spinal cord was calculated by tissue fluorescence panoramic scanning at $\times 5$ magnification. The remaining 4 random fields were observed at $\times 40$ magnification to calculate the number of Iba1+/iNOS+ cells and the number of Iba1+/Arg1+ cells. The normalized mean fluorescence intensity of GFAP, Iba1, and MMP-9 in the central canal of the spinal cord was calculated. The experimenter who performed the analysis was unaware of the experimental groups of the rats.

\section{RT-qPCR}

For quantitative real-time PCR (RT-qPCR), total RNA was first extracted from spinal cord tissues with TRIzol (Invitrogen, Thermo Fisher Scientific, Inc., USA) according to the manufacturer's protocol. The ratios of absorbance at $260 / 280 \mathrm{~nm}$ and $260 / 230 \mathrm{~nm}$ were then determined using an ultraviolet-visible light spectrophotometer (NanoDrop 2000, Thermo, Waltham, MA, USA) to assess the purity and concentration of total RNA in each sample. A FastKing cDNA First Strand Synthesis Kit (Tiangen, Beijing, China) was used to synthesize cDNA from total RNA ( $2 \mu \mathrm{g} /$ sample), and then RT-qPCR was carried out using SYBR Green PCR Master Mix (Tiangen, Beijing, China). The expression level of glyceraldehyde 3phosphate dehydrogenase (GAPDH) was used as an internal control. Three replicate wells were set up for each reaction. All primers used in this experiment were supplied by Sangon Biotech Co., Ltd. (Shanghai) (Table 1 lists the primer sequences). QuantStudio Design and Analysis Software (Applied Biosystems) was used to perform the following reaction: initial denaturation at $95^{\circ} \mathrm{C}$ for $15 \mathrm{~min}$ and $40 \mathrm{cycles}$ of $95^{\circ} \mathrm{C}$ for $10 \mathrm{~s}$, annealing at $55^{\circ} \mathrm{C}$ for $30 \mathrm{~s}$ and $72^{\circ} \mathrm{C}$ for $32 \mathrm{~s}$ (for SNCA, GAPDH, IL- $1 \beta$, and CD86) or 40 cycles of $95^{\circ} \mathrm{C}$ for $15 \mathrm{~s}$, annealing at $60^{\circ} \mathrm{C}$ for $35 \mathrm{~s}$, and $72{ }^{\circ} \mathrm{C}$ for $32 \mathrm{~s}$ (for CD206 and IL-10). Melting curve analysis confirmed the primer specificity and determined the cycle threshold (CT) fluorescence values. The data were analysed by the $2-\Delta \Delta C T$ method by researchers blinded to the experimental groups of the animals. 
Table 1 Forward and reverse primer sequences for qRT-PCR

\begin{tabular}{lll}
\hline Rat gene & Forward primer (5'-3') & Reverse primer (5'-3') \\
\hline SNCA & FP: 5'-GTGGCTGCTGCTGAGAAAAC-3' & RP: 5'-TCCATGAACGACTCCCTCCT-3' \\
GAPDH & FP: 5'-GGCACAGTCAAGGCTGAGAATG-3' & RP: 5'-ATGGTGGTGAAGACGCCAGTA-3' \\
IL-1 $\beta$ & FP: 5'-AATGCCTCGTGCTGTCTGA-3' & RP: 5'-GGATTTGTCGTTGCTTGTCTC-3' \\
CD86 & FP 5'-GATTGCAGGTCCCAGTTCACTTC-3' \\
CD206 & FP: 5' 5'-CCACTGTCCTGCTTGGACTCAC-3' \\
IL-10 & FP: 5'-CAGACCCACATGCTCCGAGA-3' & RP: 5'-GGTCAGGAGTGGTGTGGGC-3' \\
\hline
\end{tabular}

\section{Western blotting}

Total protein was extracted using radioimmunoprecipitation assay (RIPA) lysis buffer (including protease inhibitor cocktail) [15]. After measuring the protein concentration using a BCA assay kit (Thermo Scientific, MA, USA), the protein was subjected to Western blotting. Equal amounts of protein from each sample were separated using 8\%, 15\% SDS-PAGE and transferred to polyvinylidene fluoride membranes (Merck Millipore, Billerica, MA, USA). The membranes were blocked with $5 \%$ skim milk at room temperature $\left(22-25^{\circ} \mathrm{C}\right)$ for $1 \mathrm{~h}$ and then incubated with a monoclonal rabbit anti- $\alpha$-Syn antibody (1:10000, Abcam), a polyclonal mouse anti- $\alpha-$ Syn antibody (1:500, BD Biosciences), and a rabbit anti$\alpha$-Syn primary antibody (phospho-S129, 1:1000, Abcam) or a rabbit anti- $\beta$-actin antibody (1:10000; Abcam, Cambridge, UK) overnight at $4{ }^{\circ} \mathrm{C}$. After incubation with the corresponding secondary antibodies for $1 \mathrm{~h}$, the membranes were scanned using an Odyssey Sa imaging system (LI-COR Biosciences, Lincoln, NE, USA). The density of the results was quantified by two experimenters using Image software $(\mathrm{NIH}$, Bethesda, MD, USA), and the two experimenters were blinded to the characteristics of the samples studied.

\section{ELISA}

Serum samples were prepared by centrifugation using coagulant blood collection tubes. Enzyme-linked immunosorbent assay (ELISA) kits were used to measure the serum levels of TNF- $\alpha$, IL- $1 \beta$, IL-2, IL-10, IL-4, transforming growth factor- $\beta$ (TGF- $\beta$ ) (Boster Biological Technology, Ltd., Wuhan, China), and IFN gamma (IFN- $\gamma$, R\&D Systems, Minneapolis, MN) according to the manufacturer's instructions on the 28th day after surgery. The absorbance was measured at $450 \mathrm{~nm}$ with a MultiMode Microplate Reader (Varioskan Flash, Thermo Scientific Inc., USA). The concentrations of TNF- $\alpha$, IL-1 $\beta$, IL-2, IL-10, IL-4, TGF- $\beta$, and IFN- $\gamma$ were calculated from standard curves and are expressed in $\mathrm{pg} / \mathrm{ml}$.

\section{Statistical analysis}

The data are expressed as the mean \pm standard deviation (SD). Statistical analysis was performed in GraphPad Prism 7.0 (GraphPad Software Inc., San Diego, CA).
Comparisons between the two groups were performed using Student's $t$ test or the Mann-Whitney test, as appropriate. One-way and two-way analysis of variance (ANOVA) and Tukey's test for multiple comparisons were used to analyse differences between groups. BBB motor function scores were analysed by two-way repeated-measures ANOVA followed by the Sidak multiple comparisons test. A $p$ value $<0.05$ was considered to indicate statistical significance. ${ }^{*} p<0.05,{ }^{* *} p<0.01$, **** $p<0.001$, and $* * * * 0.0001$.

\section{Results}

We designed a total of 5 sets of SNCA-shRNA sequence plasmids with GFP fluorescent tags, integrated them into lentiviruses, and transfected them into HEK293 cells to observe the degree of transfection. RT-qPCR experiments were performed. The results are shown in more detail in Additional file 1. To reduce experimental error, all animals received injections of lentiviruses including the gene of interest and an empty vector. None of the animals in the experiment died due to injection of the virus. To first ensure that the impact parameters were similar between groups, we examined the IH impactor output. The animals received contusions of the central thoracic spinal cord at T3, with an average contusion pressure of $402.26 \pm 0.672$ kilodynes, which was close to the initial contused peak force of 400 kilodynes. This difference was attributable to inertial compensation. In addition, 5 animals did not survive SCI, and 3 animals did not survive due to severe bladder dysfunction after surgery. Male animals were used because the majority of SCI cases occur in males [16].

\section{Temporal and spatial expression levels of a-Syn in the spinal cord after $\mathrm{SCl}$}

The oligomerization of $\alpha$-Syn is a hallmark of chronic neurodegenerative synucleinopathies and causes its aggregation over time. In this study, we first examined the expression of $\alpha$-Syn and phosphorylated $\alpha$-Syn ( $p-\alpha-S y n$, Ser-129) in the injured site after SCI by Western blotting. The results showed that the expression of $\alpha$-Syn at the early injury site at $1 \mathrm{dpi}$ was higher in the SCI group than in the sham group, but the difference was not significant. The expression levels of $\alpha-S y n$ and $p-\alpha-S y n$ 
gradually increased from $7 \mathrm{dpi}$ to $14 \mathrm{dpi}$ and remained unchanged through $28 \mathrm{dpi}$ in the SCI group relative to the sham group (Fig. 2a-c). RT-qPCR was used to detect transcriptional changes in the SNCA gene at the SCI site and indicated a gradual increase after SCI (Fig. 2d). Statistical analysis showed that, compared with that in the sham group, the transcriptional expression of SNCA increased after injury in the SCI group, and $\alpha$-Syn and p$\alpha$-Syn protein levels increased significantly. There was a significant increase the first day after injury, and the most significant increase was observed on the 14th day.
Second, the expression of $\alpha$-Syn in the GM and WM of the spinal cord was detected by IHC on the 28th day after SCI. Analysis of the mean IOD revealed that the expression of $\alpha$-Syn in the WM was significantly higher in the SCI group than in the sham group $(p<0.0001)$ and showed a scattered punctate distribution (Fig. 2e, g). The GM expression was significantly higher in the SCI group than in the sham group ( $p=0.023$ ) (Fig. 2e, f).

To investigate the role of $\alpha$-Syn in the spinal cord, we used a lentiviral vector to knock down the expression of SNCA in the injured area immediately after SCI. To confirm whether

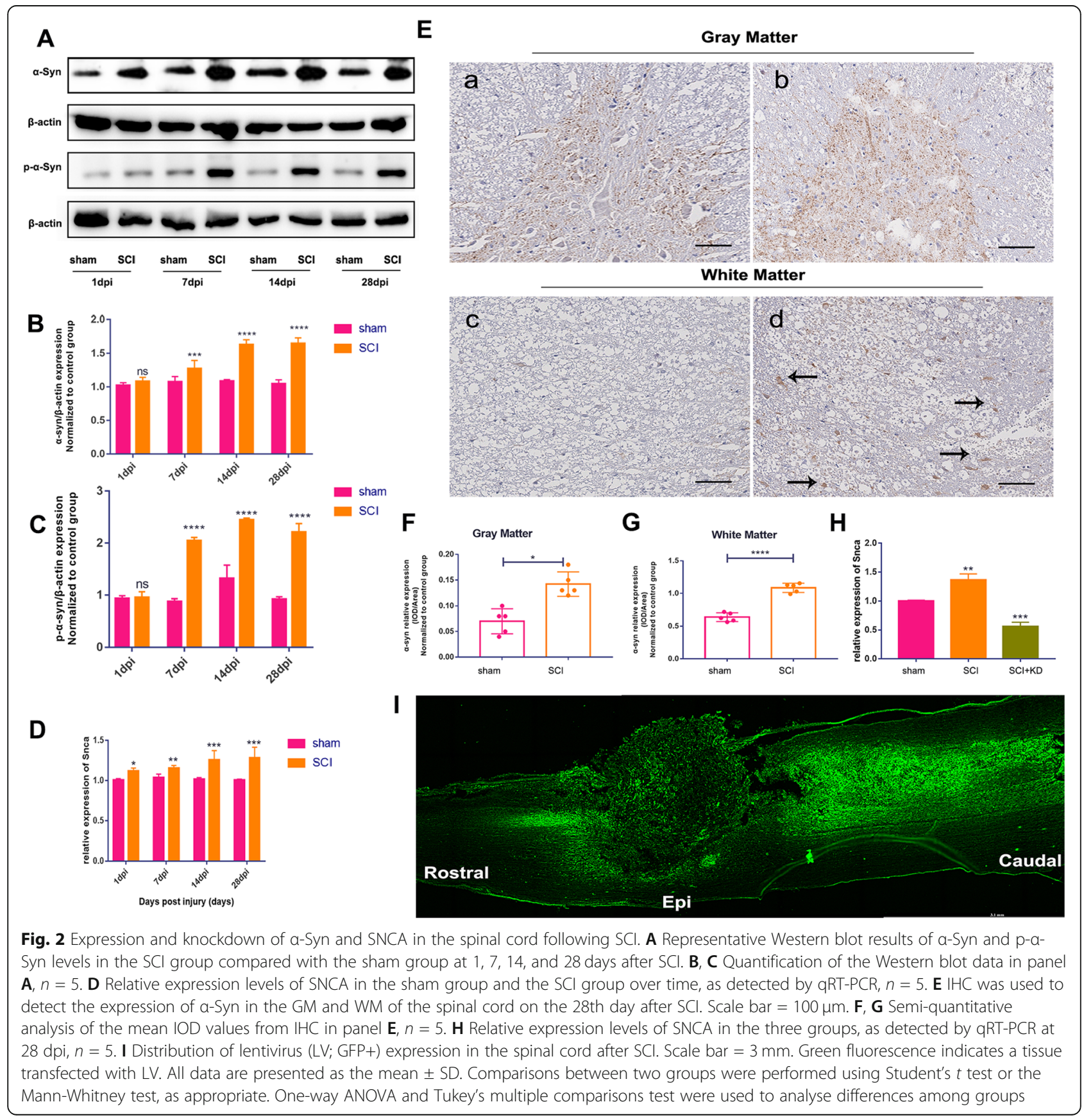


the lentivirus transduced the target tissue, we immediately injected a lentiviral GFP vector (LV-GFP; $10 \mu \mathrm{l} ; 1.0 \times 10 \mathrm{E} 9$ $\mathrm{TU} / \mathrm{ml}$ ) into the injury sites of the rats. We examined spinal cord sections at $7 \mathrm{dpi}$ and found that the lentivirus successfully transduced the spinal cord at the site of injury (Fig. 2i). Next, RT-qPCR was performed to verify the expression of SNCA in the sham group, $\mathrm{SCI}$ group, and $\mathrm{SCI}+\mathrm{KD}$ group at 28 dpi. The SCI+KD group exhibited significantly lower expression than the SCI group, as shown in Fig. $2 \mathrm{~h}$.

\section{Blocking a-Syn improves neurological outcomes and functional recovery after $\mathrm{SCl}$}

We next assessed the therapeutic effect of $\alpha$-Syn blockade after SCI. We used BBB motor function scores and footprint analysis to assess motor function for 28 days after SCI. Normal motor function was scored as 21 points. The $\mathrm{SCI}$ and $\mathrm{SCI}+\mathrm{KD}$ groups exhibited scores significantly lower than those in the sham group $(p<0.0001)$. As shown by the $\mathrm{BBB}$ exercise scale scores, no significant differences were found between the $\mathrm{SCI}$ group and the $\mathrm{SCI}+\mathrm{KD}$ group early after SCI $(1,3,7,10$, and $14 \mathrm{dpi})$; the $\mathrm{SCI}+\mathrm{KD}$ group scores were comparable with the SCI group scores $(p=$
0.99 (1 dpi), $p=0.97$ (3 dpi), $p=0.49$ (7 dpi), $p=0.07$ (10 $\mathrm{dpi})$, and $p=0.07(14 \mathrm{dpi}))$. As the observations continued, we noted better functional recovery in the $\mathrm{SCI}+\mathrm{KD}$ group than in the SCI group, as indicated by better scores $p=$ 0.01 (21 dpi) and $p=0.02(28 \mathrm{dpi})$ ) (Fig. 3a). Figure 3b shows the significant difference in the recovery of motor function at the end of observation on day 28.

In the footprint analysis, the LV-SNCA-shRNA-treated SCI rats showed relatively consistent hindlimb coordination (blue ink) 28 days after SCI compared with the empty vector-treated $\mathrm{SCI}$ rats. The $\mathrm{SCI}+\mathrm{KD}$ group exhibited a gait more similar to that of the sham group. In contrast, $\mathrm{SCI}$ animals receiving empty vector treatment showed incongruous gaits and extensive drag of both hindlimbs, as indicated by ink streaks (Fig. 3c). These data indicate that downregulation of $\alpha$-Syn after SCI improves motor function in SCI rats.

\section{Downregulation of a-Syn after $\mathrm{SCl}$ can ameliorate pathological findings in injured tissue}

We examined the expression of GFAP and Iba1 around the injury site on the first post-operative day by IHC. There was no significant difference between the SCI

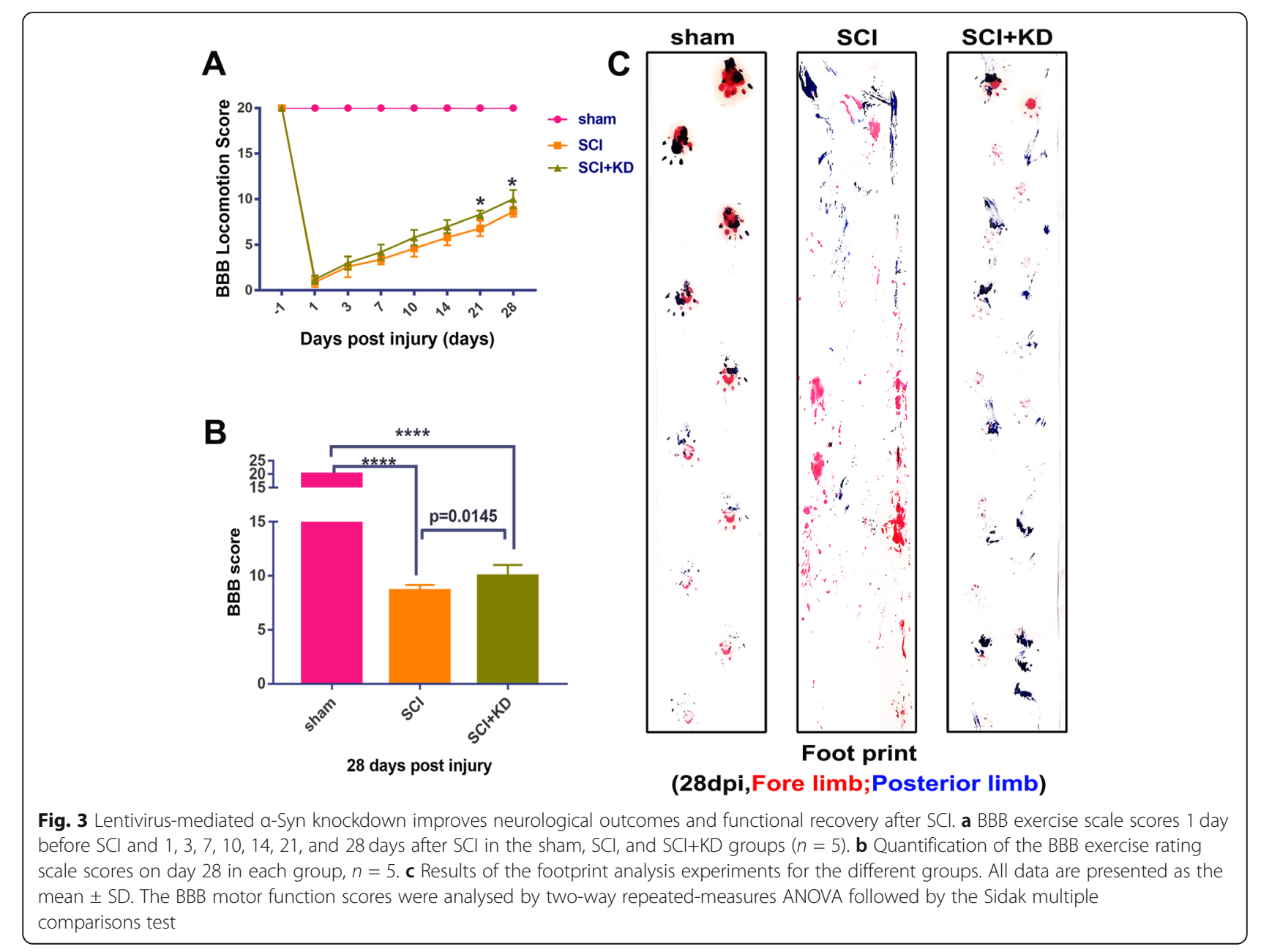


group and the $\mathrm{SCI}+\mathrm{KD}$ group; however, we found that compared with the sham group, the other two groups exhibited significant increases in glial cell numbers and activity, very obvious changes in microglial morphology, retraction of cell protrusions, and obvious enlargement and thickening/clustering of the cell body (see Additional file 2: Figure S3). In summary, glial cell activation immediately after injury may be associated with preformed $\alpha$-Syn. We also assessed the extent of tissue preservation 7 days after SCI. We performed morphometric analyses of LFB and HE staining of paraffinembedded spinal cord tissue and made serial sections of the tissue $4 \mathrm{~mm}$ away from the centre of the injury. Cross-sections of tissues taken from areas different distances from the injury site ( 2 to $4 \mathrm{~mm}$ from the centre of the lesion in the rostral-caudal plane) were quantified. LFB staining was used to observe the loss of myelin in sections from the middles of the two ends of the spinal cord (Fig. 4a,c). In addition, we performed HE staining to observe the infiltration of blood cells (dark red cells) in sections from the middles of the two ends of the spinal cord and the surrounding lesions (Fig. 4b). The staining objectively showed that infiltration of large amounts of peripheral blood above and below the level of the SCI, indicating destruction of the BCSB. Compared with the $\mathrm{SCI}+\mathrm{KD}$ group, the $\mathrm{SCI}$ group showed a greater amount of peripheral blood infiltration, suggesting that the BCSB functional damage was more severe.

At the distal end of the injured spinal cord, there was a large demyelinated cavity near the central canal, and there were large and small demyelinated lesions in the WM accompanied by peripheral blood cell infiltration, which are pathological features of secondary injury. In addition, the SCI+KD group exhibited reduced myelin loss and peripheral blood cell infiltration in the distal end of the spinal cord.

\section{Reduced a-Syn levels can reduce microglial numbers and activation}

Microglia and astrocytes are the main effectors of neuroinflammation after injury $[17,18]$. To analyse these cells, we performed IF staining of microglia (Iba1) in frozen sections on the 28th day after SCI (Fig. 5a). Panoramic scanning of tissue sections was performed under a $\times 5$ objective, and the numbers of microglia before and after injury were compared. The number of Iba1+ cells in the SCI group was significantly higher than that in the $\mathrm{SCI}+\mathrm{KD}$ group, and the numbers in both groups were significantly higher than that in the sham group (Fig. 5b). High-power microscopy showed that the sham group mainly contained a small number of highly branched microglia (Fig. 5c). The SCI group and the $\mathrm{SCI}+\mathrm{KD}$ group showed considerable branch retraction, thickening, amoeba-like morphology, and peripheral blood infiltration of macrophages. The $\mathrm{SCI}+\mathrm{KD}$ group showed less microglial activation and macrophage infiltration than the SCI group.

\section{Reductions in a-Syn promote M1-to-M2 phenotypic polarization of microglia/macrophages}

Activated M1-type microglia release NO after traumatic and ischaemic injury to the CNS, and this initiates oxidative stress and a neurotoxic cascade in neurons. In addition, in M2-type microglia, arginase1 (Arg1) is known to block uncoupling of nitric oxide synthase (NOS) [19]. We observed Iba1+/iNOS+ double-labelled microglia by IF at $28 \mathrm{dpi}$. The number of these doublelabelled microglia was significantly lower in the SCI+KD group than in the SCI group but was still higher in the $\mathrm{SCI}+\mathrm{KD}$ group than in the sham group (Fig. $6 \mathrm{a}, \mathrm{b}$ ). The number of Iba1+/Arg1+ double-labelled cells was significantly higher in the $\mathrm{SCI}+\mathrm{KD}$ group than in the SCI group and the sham group (Fig. 7a, b). This finding indicates that after SCI, reductions in $\alpha$-Syn contribute to increased expression of Iba1+/Arg1+ M2-type cells, decrease the expression of Iba1+/iNOS+ M1-type cells, and promote the polarization of M1 microglia/macrophages to the M2 phenotype.

In addition, using RT-qPCR, we further tested transcriptional levels in M1/M2 microglia/macrophages in the injury site after knocking down $\alpha$-Syn expression after SCI. The relative mRNA expression levels of CD86, CD206, and the cytokines IL-1 $\beta$ and IL-10 were detected in the injured tissues on day 28 , and the changes in the pro-inflammatory (M1) response and anti-inflammatory (M2) response at the transcriptional level were observed. Among these changes, the expression of the proinflammatory cytokine IL- $1 \beta$ and the M1-type microglial/macrophage protein $\mathrm{CD} 86$ in the $\mathrm{SCI}+\mathrm{KD}$ group was significantly decreased (Fig. 6c, d). In contrast, the expression of the anti-inflammatory cytokine IL-10 and the M2-type microglial/macrophage protein CD206 in the $\mathrm{SCI}+\mathrm{KD}$ group was elevated (Fig. 7c, d). All of the above results show that reductions in $\alpha$-Syn promote the polarization of M1 microglia/macrophages to the M2 phenotype and promote the pro-inflammatory response over the anti-inflammatory response.

\section{Silencing a-Syn improves the immune microenvironment after $\mathrm{SCl}$}

The early recruitment of microglia/macrophages after $\mathrm{SCI}$ is characterized by the release of pro-inflammatory cytokines that cause cell death and tissue degeneration following injury [20]. ELISA was used to analyse the expression of the pro-inflammatory cytokines TNF- $\alpha$, IL-1 $\beta$, IL-2, and IFN- $\gamma$ (Fig. 8a-d) and the antiinflammatory cytokines IL-10, IL- 4 , and TGF- $\beta 1$ in the serum of each group on the 28th day after the operation. 

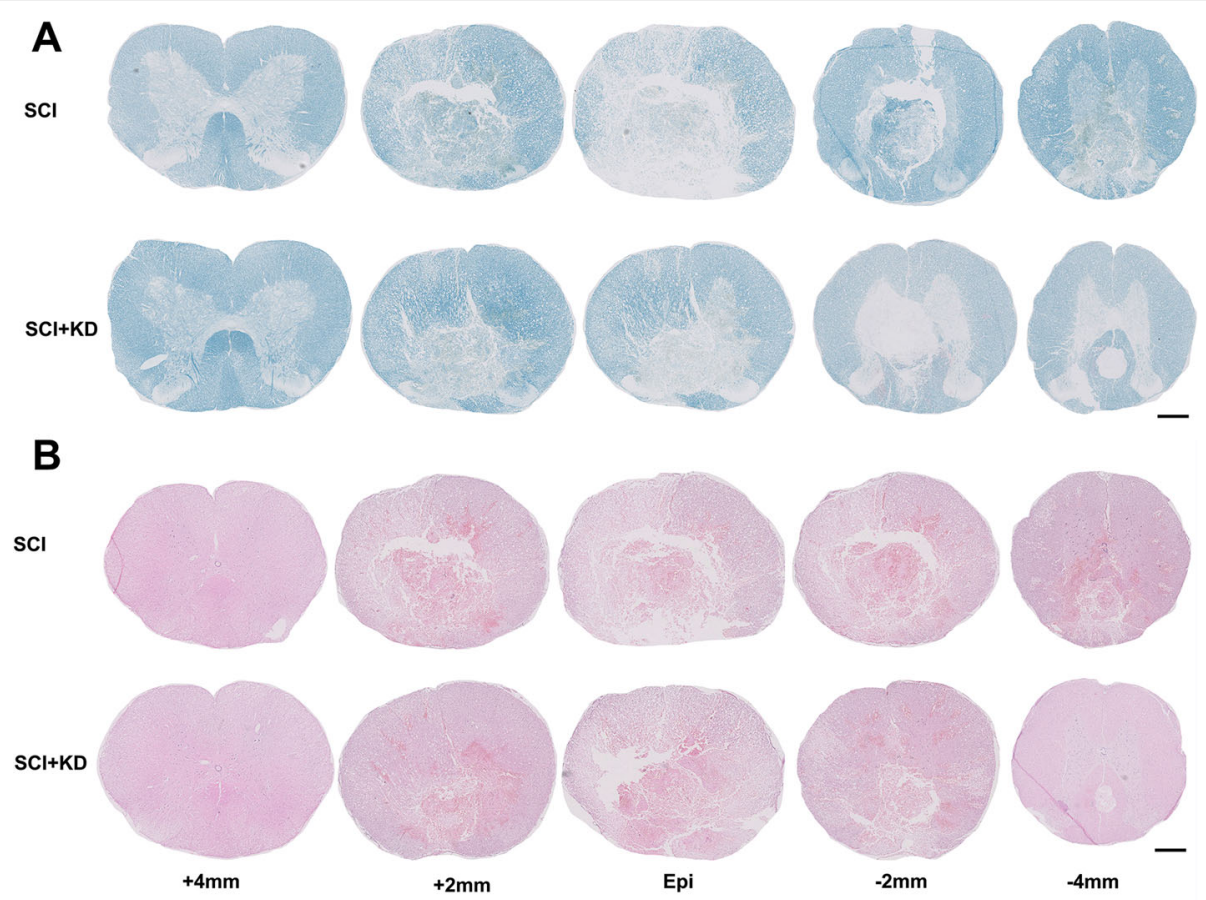

C

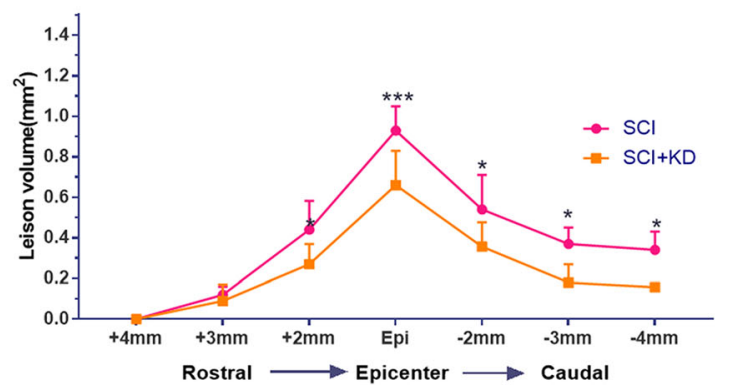

Fig. 4 Downregulation of a-Syn after SCl can ameliorate pathological findings in injured spinal cord tissue. a Representative images showing LFB staining after SCl. Scale bar $=1 \mathrm{~mm}$. b Representative images showing HE staining after SCl. Scale bar $=1 \mathrm{~mm}$. c Quantitative analysis of the results in panel $\mathbf{a}$. All data are presented as the mean $\pm \mathrm{SD}, n=5$. For staining that was performed $2 \mathrm{~mm}$ from both ends in the $\mathrm{SCl}+\mathrm{KD}$ group and the SCl group, $p<0.05$; for staining that was performed at the centre of the $\mathrm{SCl}$ area in the $\mathrm{SCl}+\mathrm{KD}$ group, $p<0.001$ compared with that in the SCI group. One-way ANOVA and Tukey's multiple comparisons test were used to analyse differences among groups

The levels of the anti-inflammatory cytokines IL-10, IL-4, and TGF- $\beta 1$ in the SCI+KD group were higher than those in the SCI group, and the expression of IL-10 was significantly higher than that in the SCI group (Fig. 8e-g). The results show that targeting the knockdown of $\alpha$-Syn promotes the transformation of peripheral blood from a proinflammatory to an anti-inflammatory state after SCI, thereby improving the immune microenvironment to allow neuronal survival and reduce secondary damage.

\section{Decreases in a-Syn can reduce the activation of microglia/astrocytes in the central canal of the spinal cord after $\mathrm{SCl}$}

In the above experiment, considerable peripheral blood cell infiltration and demyelination in the cavity around the central canal of the spinal cord were observed after
SCI. Therefore, we next evaluated the effect of reducing $\alpha$-Syn on microglial/astrocyte proliferation in the central spinal canal 28 days after SCI. It is currently believed that astrocytes exist in three states, namely, a resting state, an activated state, and a proliferative state. Reactive astrocyte proliferation is mainly identified by increased expression of GFAP [21].

Evaluation of GFAP+/Iba1+ double staining in SCI lesions showed that the astrocytes in the SCI group and the $\mathrm{SCI}+\mathrm{KD}$ group were hypertrophied, and their branches were thickened, showing morphological changes associated with reactive astrocytes. Compared with that in the $\mathrm{SCI}$ group, the average fluorescence intensity of GFAP in the $\mathrm{SCI}+\mathrm{KD}$ group was downregulated at 28 days, but the difference was not significant $(p=0.16)$. The GFAP signals remained elevated (1- to 1.5 -fold) in the $\mathrm{SCI}+\mathrm{KD}$ group 


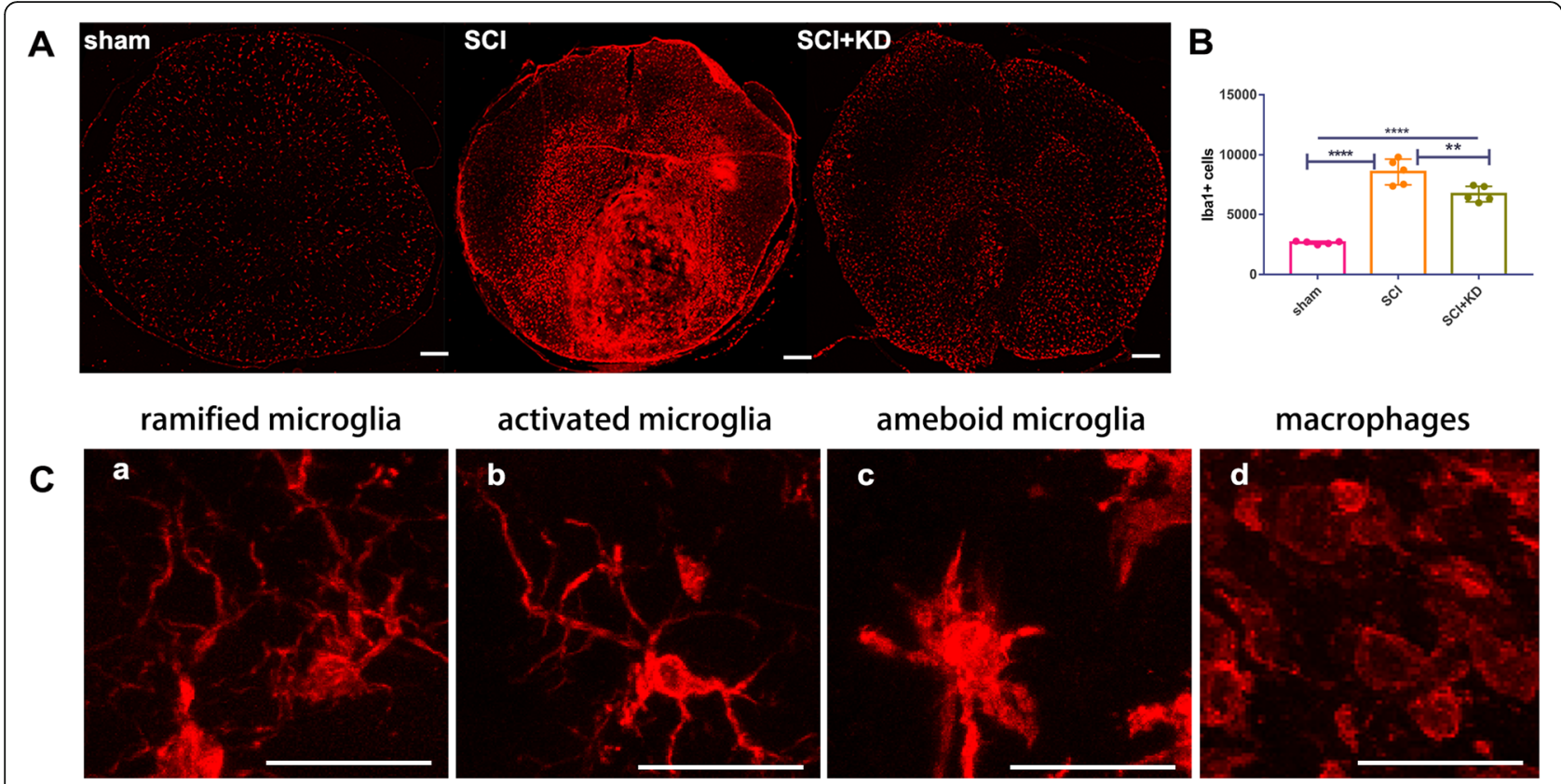

Fig. 5 Reduced levels of a-Syn can reduce the microglial numbers and activation. A Representative images of Iba1 IF staining after $\mathrm{SCl}$. Scale bar $=1$ $\mathrm{mm}$. B Quantitative analysis of the results in panel A. All data are presented as the mean $\pm S D, n=5$. One-way ANOVA and Tukey's multiple comparisons test were used to analyse differences among groups. The $p$ values for the $\mathrm{SCl}+\mathrm{KD}$ and $\mathrm{SCl}$ groups compared with the sham group were $p<0.05$ and $p<0.001$, respectively. C Under high magnification, four different typical forms of Iba1+ cells were observed

compared with the sham group ( $p<0.05, n=5$ /group) (Fig. 9a, b). Consistent with our above experimental data, knockdown of $\alpha$-Syn, compared with empty vector treatment, significantly attenuated microglial signals in the spinal cord central canal at $28 \mathrm{dpi}(p<0.05, n=5$ /group $)$ (Fig. 9a, c). Importantly, we observed tight junctions between microglia and astrocyte branches through 3D image visualization, and the SCI group showed closer connections than the other groups. These findings indicate that $\alpha$-Syn knockdown can preferentially reduce microglial activation, reduce astrocytic density, and further reduce reactive astrocyte activation and neuroinflammation near the central canal of the spinal cord.

\section{Knocking down a-Syn can reduce the expression of MMP-}

\section{9 in the central canal of the spinal cord}

Since we determined that reducing $\alpha$-Syn attenuates pro-inflammatory mediator levels and neuroinflammatory responses, we next examined whether this change inhibits the activity of matrix metalloproteinases (MMPs) after SCI. MMP-9 plays a major role in tissue degeneration and reconstruction after injury [22, 23]. GFAP+/MMP-9+ double staining was analysed by IF. Compared with that in the SCI group, the expression of MMP-9 in injured spinal cord tissue in the SCI+KD group was decreased on the 28th day after injury $(p<$ $0.05, n=5$ /group) (Fig. 8a, b). Studies have shown that MMP-9 and astrocytes are involved in the homeostasis and maintenance of the BCSB and that elevated MMP-9 expression leads to endothelial cell dysfunction [24]. Our results indicate that knockdown of $\alpha$-Syn after SCI can reduce MMP-9 expression in the central canal of the spinal cord (Fig.10A-b, f, j). As shown in Fig. 10Ad, h, i, MMP-9 was expressed along the endothelial cells of the spinal cord, and astrocytes were distributed around the MMP-9-expressing cells. Increased expression of MMP-9 enhances the permeability of the BCSB, promotes the infiltration of peripheral inflammatory cells, and aggravates the development of neuroinflammation after SCI. Knocking down $\alpha$-Syn expression contributes to reductions in MMP-9, thereby reducing peripheral blood infiltration and inflammation.

\section{Hypothesis of the role of a-Syn in neuroinflammation after $\mathrm{SCl}$}

We observed increased $\alpha$-Syn expression, microglial/ astrocyte activation, peripheral blood cell infiltration, severe BCSB dysfunction, and M1-type microglial/macrophage transformation accompanied by considerable proinflammatory cytokine release secondary to $\mathrm{SCI}$, which resulted in a severe loss of distal myelin at the proximal end of the injured area. We knocked down $\alpha$-Syn expression with LV-SNCA-shRNA, which ameliorated the dysfunction and pathology, reduced CNS inflammation, and promoted anti-inflammatory responses. Furthermore, $\alpha$-Syn knockdown improved BCSB function and 


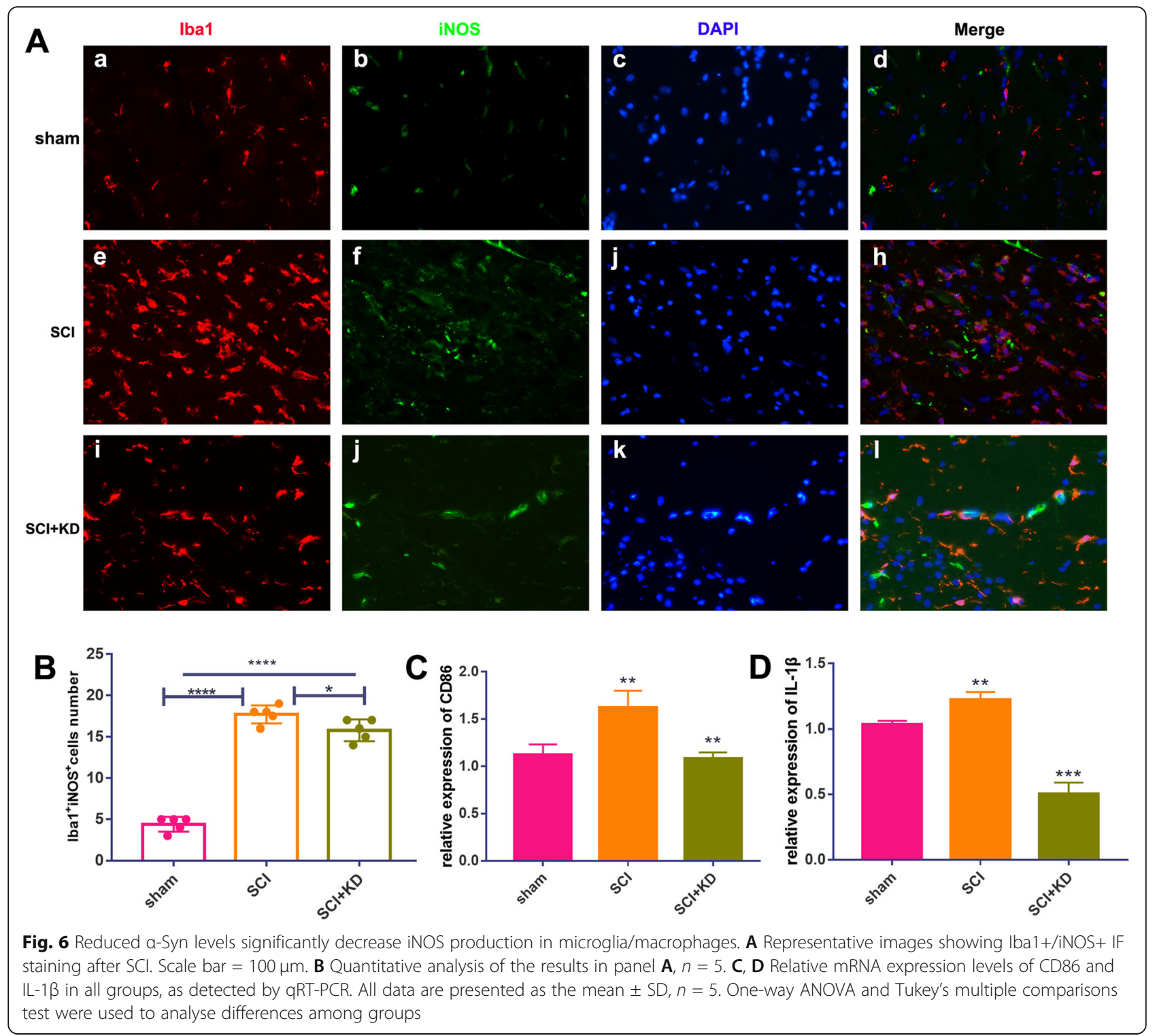

restored the immune microenvironment, which conferred neuroprotection after SCI, as shown in Fig. 11.

\section{Discussion}

We generated a Sprague-Dawley rat model of spinal cord contusion injury of the T3 segment. Injuries to high-level segments are clinically more common than injuries to lower segments, and compared with other models, contusion of $\mathrm{T} 3$ is safer with a higher survival rate [25]. Secondary SCI lesions develop within seconds to months after the primary phase and have been the focus of a series of modern medical interventions. In the present study, we showed that the expression of $\alpha$-Syn and $p-\alpha$-Syn (Ser129) was significantly increased in the early stage of SCI. In addition, the increased expression was mainly concentrated in the WM of the spinal cord with a scattered distribution. LV-SNCA-shRNA transfection knocked down spinal $\alpha$-Syn early after SCI. We found that $\alpha$-Syn is an important promoter of neuroinflammatory factors. During secondary injury after SCI, $\alpha$-Syn reduced the activation of microglia/astrocytes and the expression of iNOS and promoted the transformation of M1 macrophages to the M2 phenotype, and these changes were accompanied by increases in Arg1 and IL-10 expression. Significant increases in the expression of these cytokines regulate neuroinflammation in the spinal cord. The SCI+KD group also showed decreases in CD86+ M1-type microglia and IL-1 $\beta$ levels and an elevated CD206+ M2 phenotype at the transcriptional level. The peripheral blood serum levels of IL-1 $\beta$, TNF- $\alpha$, and IL-2 were also decreased, and the levels of the anti-inflammatory cytokine IL-10 were significantly 


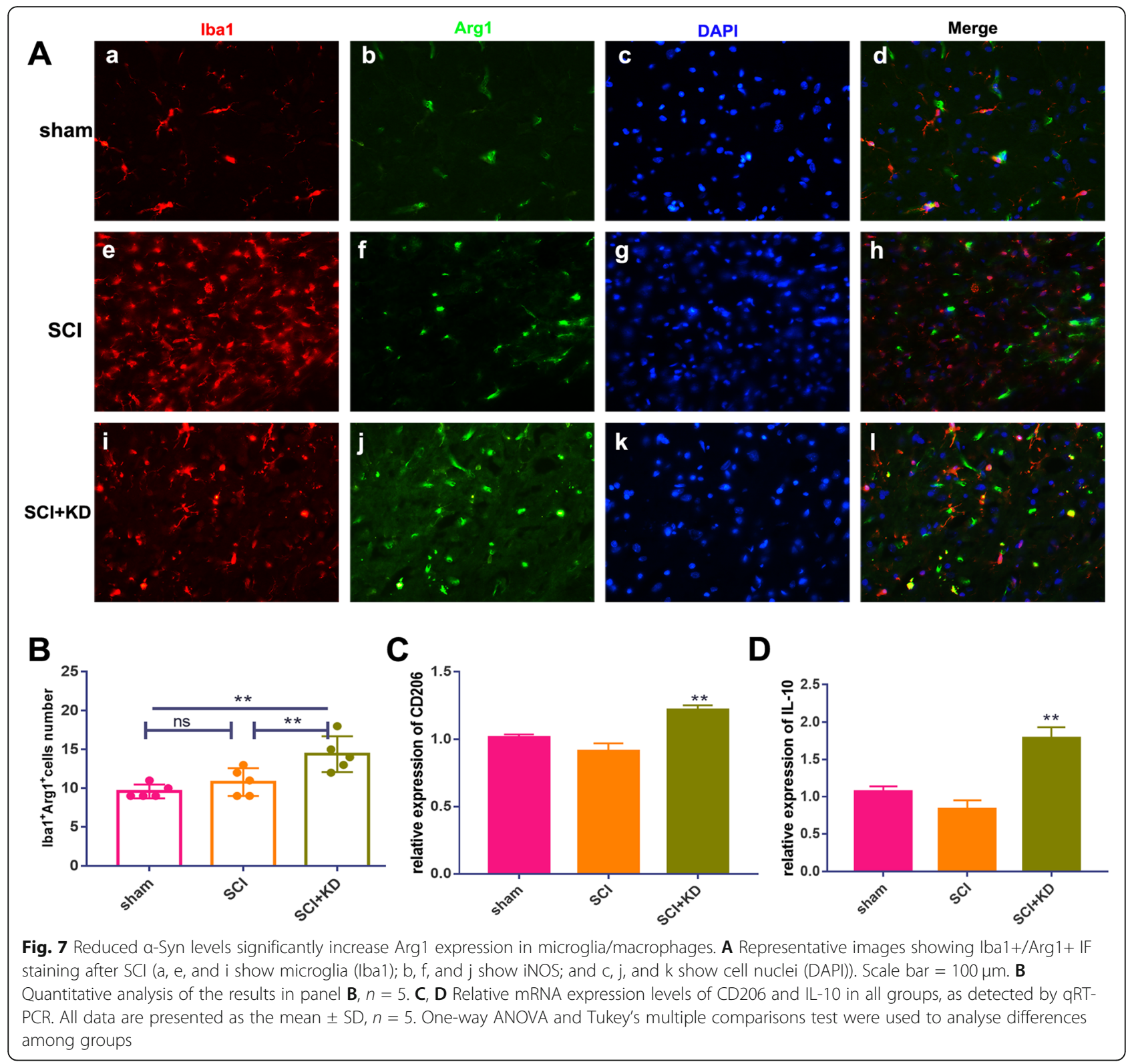

increased. In addition, we found that the $\mathrm{SCI}+\mathrm{KD}$ group exhibited reduced expression of MMP-9, which may have improved the function of the BCSB, a critical barrier for central immunity and peripheral immune stabilization.

The $\alpha$-Syn protein encoded by the SNCA gene is a small (14-kD) presynaptic nerve terminal protein [26-28]. Intracellular aggregation of this 140-amino-acid neuronal protein is thought to play an important role in the pathogenesis of neurodegenerative diseases, including Parkinson's disease (PD) and Alzheimer's disease (AD) [29, 30]. Over the last decade, genome-wide association studies (GWASs) and candidate gene-based approaches have implicated SNCA mutant-type as a highly significant genetic risk factor for synucleinopathies [55]. A study by Sakurai et al. investigated biomarkers of SCI, specifically DJ-1,
PINK1, and $\alpha$-Syn, in rabbits. After transient spinal cord ischaemia, $\alpha$-Syn participates in spinal motor neuron death, possibly through oxidative stress [31]. Another study by Busch et al. revealed that SNCA accumulation is prioritized and is correlated closely with an increased risk of neuronal death after SCI [32]. A study by Kim et al. showed that transient focal cerebral ischaemia upregulates $\alpha$-Syn protein expression and nuclear translocation in adult rodent brain neurons and that knockdown of $\alpha$-Syn reduces cerebral infarct area, mitochondrial rupture, oxidative stress, apoptosis, and autophagy and promotes better neurological recovery after cerebral ischaemia [33]. These results indicate that abnormal aggregation of $\alpha$-Syn promotes neuronal death not only in the contexts of chronic neurodegenerative diseases such as PD but also in 
A

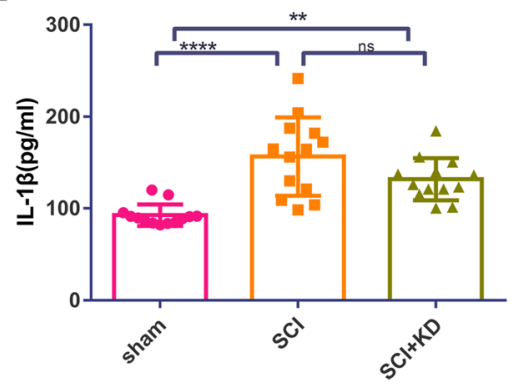

C

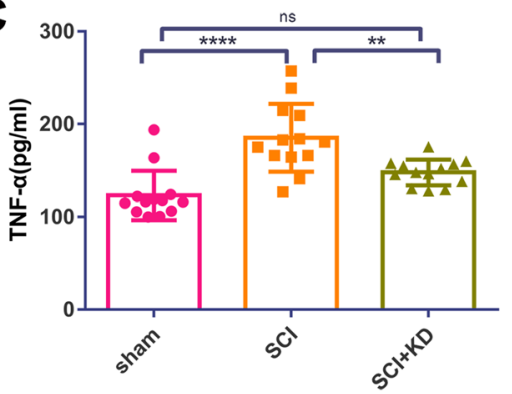

E

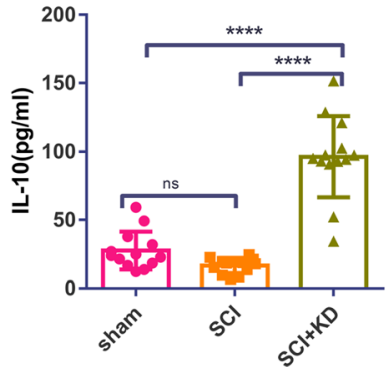

$F$

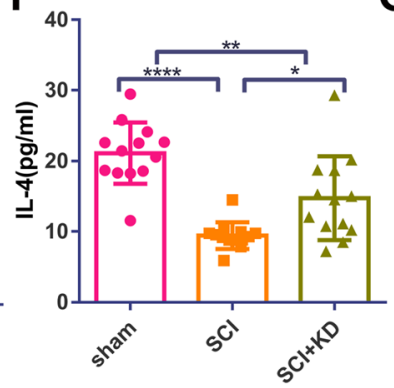

B

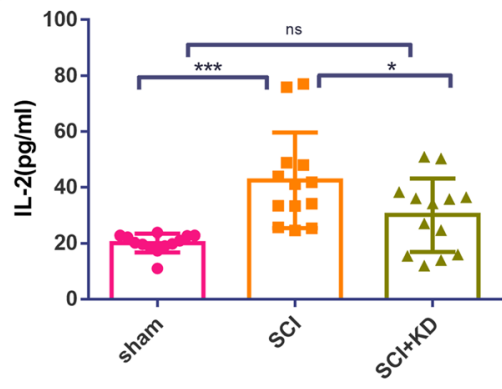

D

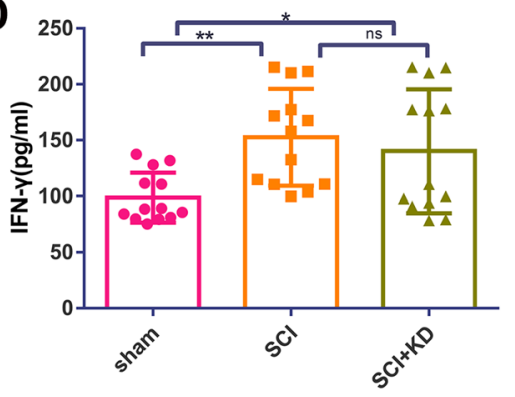

G

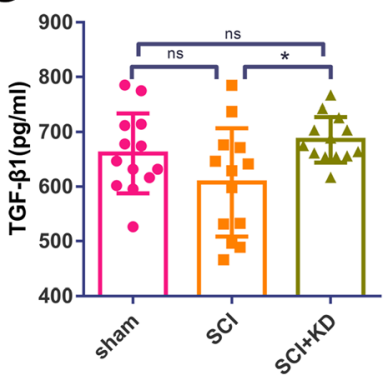

Fig. 8 Knockdown of a-Syn promotes changes in peripheral serum inflammation after SCl. a-d Changes in the levels of the pro-inflammatory cytokines IL-1 $\beta, I L-2$, TNF- $\alpha$, and IFN- $\gamma, n=13$. e- $\mathbf{g}$ Changes in the levels of the anti-inflammatory cytokines IL-10, IL-4, and TGF- $\beta 1, n=13$. All data are presented as the mean \pm SD. One-way ANOVA and Tukey's multiple comparisons test were used to analyse differences among groups

ischaemic lesions. In another recent study, miR-7 was shown to attenuate $\alpha$-Syn-mediated secondary brain injury after stroke, possibly by acting on multiple downstream targets of apoptosis, autophagy, oxidative stress, and mitochondrial damage, and to prevent neuropathology associated with stroke [34]. In addition, various animal studies have identified the ability of $\alpha$-Syn to induce apoptotic gene expression, thereby promoting neuronal death in vivo $[35,36]$. Notably, Feng et al. showed that downregulation of SNCA promotes ciliary neurotrophic factor (CNTF) expression, inhibits neuronal apoptosis, and promotes nerve regeneration [9]. In addition to in vitro and animal models, patients with CNS injury also exhibit elevated levels of $\alpha$-Syn in the cerebrospinal fluid and axons [37-40]. Population-based studies have also shown increased incidences of neurodegenerative diseases (including PD and $\mathrm{AD}$ ) in patients with previous SCIs [41, 42].

Abnormal deposition of proteins, including amyloid- $\beta, \alpha-$ Syn, and prions, wshich can easily cause neuroinflammation, not only directly occurs in neurons but also involves glial cells [43]. $\alpha$-Syn is involved in neuroinflammation and subsequent apoptosis in the CNS. However, to date, only a few studies (primarily in vivo studies) have investigated the pathophysiological effects of $\alpha$-Syn on affected nerve tissue after SCI. A recent study by Qiao et al. found a role for $\alpha$ Syn (SNCA) in microglial migration [12]. Another study by this team isolated primary microglia from Sprague-Dawley rats and exogenously exposed them to three different doses of $\alpha$-Syn oligomers, finding that $\alpha$-Syn stimulates toll-like receptor 2 (TLR2) by inducing microglial activation after SCI [44]. These findings indicate that $\alpha$-Syn is an important inducer of neuroinflammation and concurrent neuronal loss after SCI. Consistent with our experimental results, it has been determined that $\alpha$-Syn is closely related to neuroinflammation after SCI. Microglia can exhibit an M1 or M2 phenotype based on pro-inflammatory or anti-inflammatory properties, respectively. Microglia that produce high levels of the pro-inflammatory factors iNOS, IL-2, IFN- $\gamma$, TNF $\alpha$, IL- 

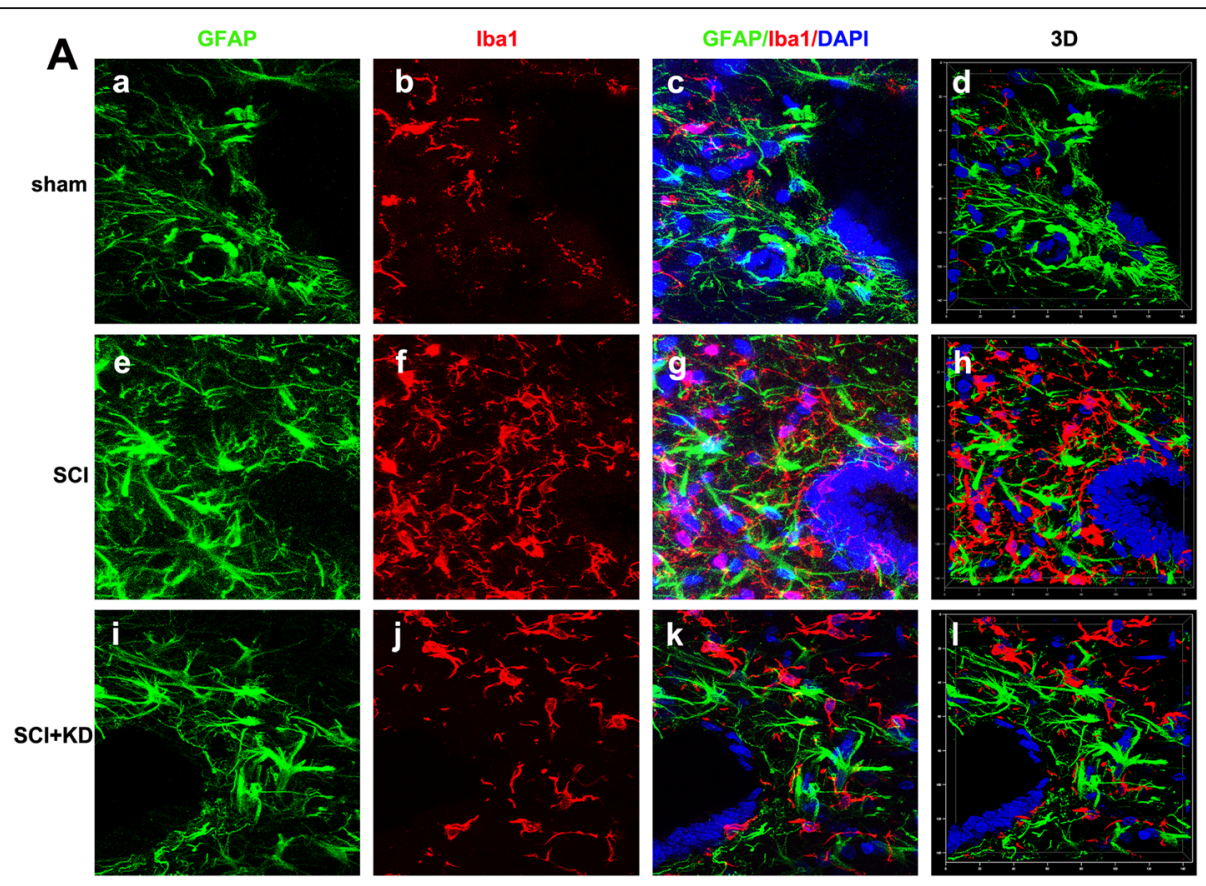

B

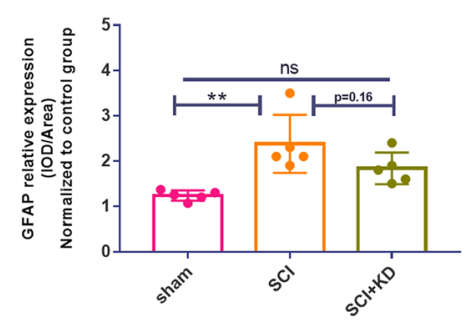

C

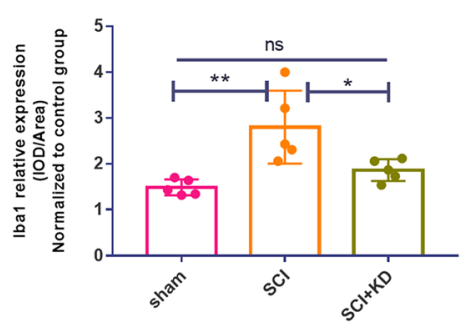

Fig. 9 Reductions in a-Syn can reduce microglial/astrocyte activation in injured spinal cord tissue. A Representative images showing GFAP+/ Ibal+ double staining after SCI by IF (a, e, and i show microglia (Iba1); b, f, and j show microglia (Iba1); c, j, and k show nuclei (DAPI); and d, h, and $\mathrm{i}$ show the 3D mode). Scale bar $=50 \mu \mathrm{m}$. B Quantitative analysis of the mean fluorescence intensity of GFAP in panel A. C Quantitative analysis of the mean fluorescence intensity of Iba1 in panel $\mathbf{A}$. All data are presented as the mean $\pm \mathrm{SD}, n=5$. One-way ANOVA and Tukey's multiple comparisons test were used to analyse differences among groups

$1 \beta$, and IL-12 are generally considered to be proinflammatory (M1-type). On the other hand, microglia that show increased levels of Arg1, CD206/Mrc1, Fizz1, Ym1, and IL-10 exhibit more of an anti-inflammatory (M2-type) phenotype [45]. A study on a PD model showed that overexpression of neuronal $\alpha$-Syn results in increases in NADPH oxidase activity, OX-6+ microglial cell numbers, and phagocytic activity marker (CD68) levels. Inflammatory M1 microglial/macrophage phenotype markers, such as iNOS, TNF- $\alpha$, IL-1 $\beta$, and IL-6, are also elevated [46]. In our study, knockdown of $\alpha$-Syn promoted the conversion of M1 microglia/macrophages to the M2 phenotype, and this effect was accompanied by increased expression of Arg1. Further research is needed to elucidate the factors that mediate microglia or peripheral macrophage activity in the context of $\mathrm{SCI}$ and to determine the exact mechanisms of action of these cells.
Another report indicated that in PD, $\alpha$-Syn induces peripheral congenital immunity followed by MHC-II adaptive immunity and changes in T-helper lymphocyte subsets [47]. Elevated neuronal $\alpha$-Syn levels promote microglial activation following spinal cord ischaemia/reperfusion injury, a process that is mediated by TLR2, but not TLR3 or TLR4, cell activation and activation of the p38 MAPK and NF-kB pathways [48].

Reactive astrocytes that express GFAP are important effectors of neuroinflammation. We detected increased numbers of astrocytes around the central canal in the surviving tissue at the injured site after $\mathrm{SCI}$, although no significant differences were detected. However, we found that astrocytes were hypertrophic after injury, which is characteristic of activated astrocytes. Under acute injury stress, astrocytes first change their cell morphology and can then release pro-inflammatory factors such as cytokines, prostaglandins, neurotrophic factors, and neuromodulators 


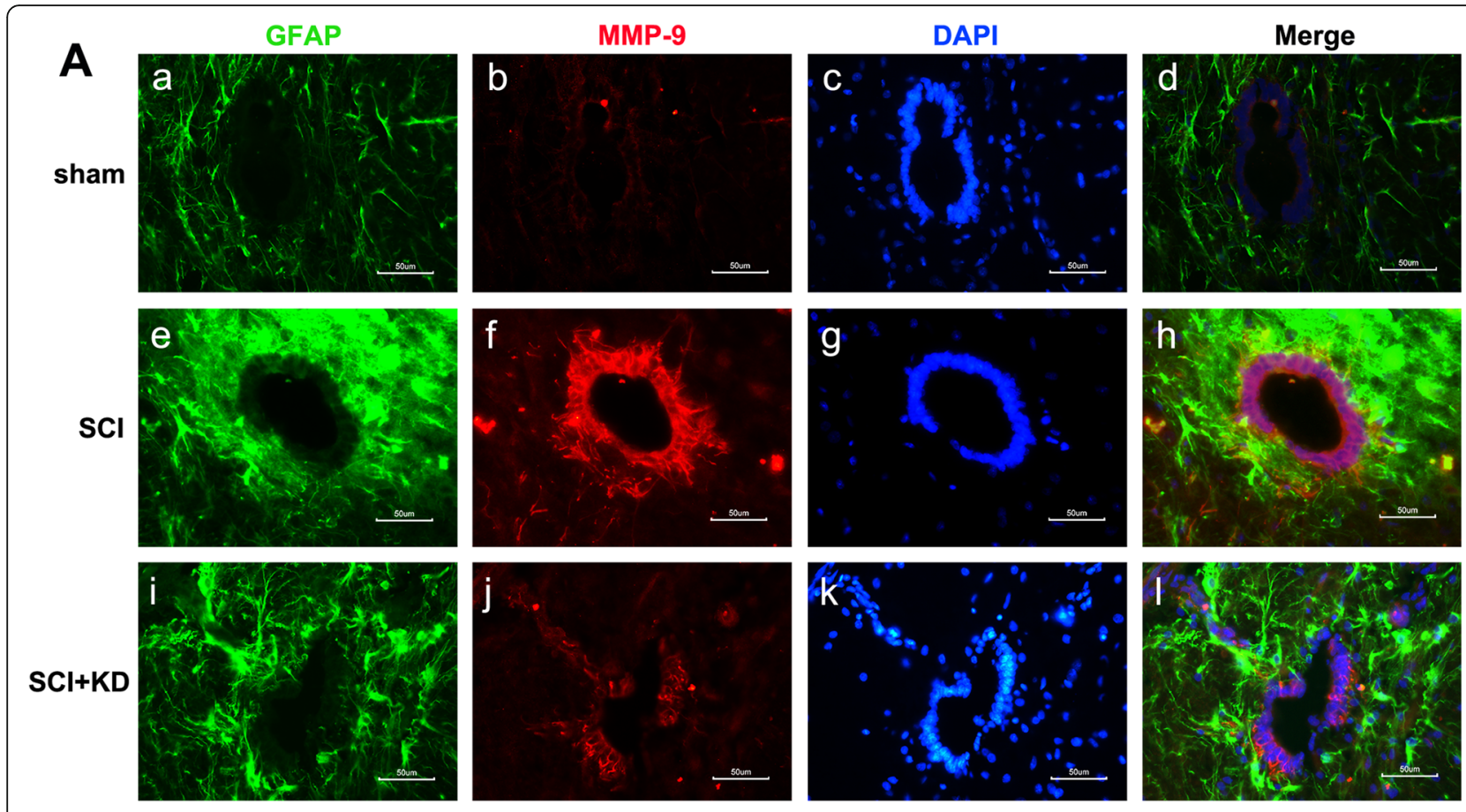

B

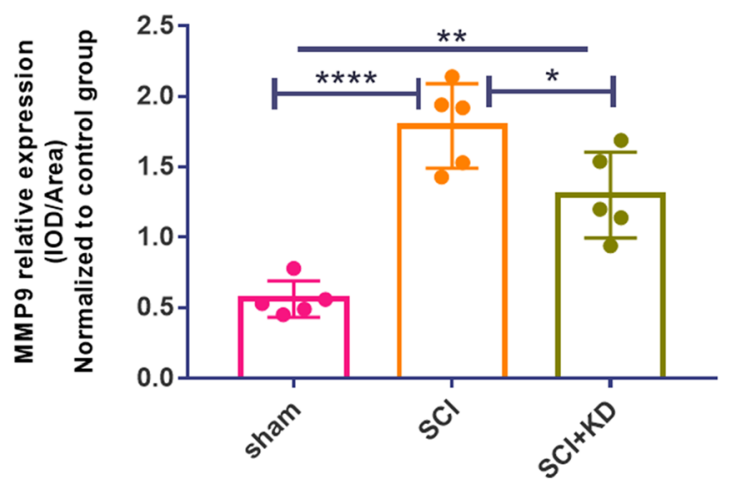

Fig. 10 a-Syn can reduce the expression of MMP-9 in the central canal of the spinal cord.A Representative images showing GFAP+/MMP-9+ double staining by IF after SCl. Scale bar $=50 \mu \mathrm{m}$. B Quantitative analysis of the mean fluorescence intensity of MMP-9 in panel A. All data are presented as the mean $\pm S D, n=5$. One-way ANOVA and Tukey's multiple comparisons test were used to analyse differences among groups

(e.g. ATP and NO), which may indicate enhancement of defence capabilities. However, most studies have shown that, with long-term sustained activation, reactive astrocytes eventually transform into glial scars that impede axonal growth in the chronic phase [49].

The highly precise function of the BCSB allows endothelial cells to tightly regulate the stability of the CNS. This structure plays a key role in maintaining the normal functions of neurons and in protecting the CNS from toxins, pathogens, inflammatory factors, and diseases. Destruction of the BCSB is an important pathophysiological basis for secondary injury after SCI. Increased MMP-9 activity is associated with several pathologies in the context SCI, including enhanced astrocyte migration, glial scar formation [50], and the destruction of the BCSB. MMP-9-deficient mice are less sensitive to BCSB destruction than wild-type mice, and this decrease in sensitivity reduces neutrophil infiltration and functional recovery after SCI [51]. We found that rats subjected to SCI and transfected with LV-SNCAshRNA exhibited reduced MMP-9 expression in the central canal of the spinal cord and reduced spinal cord haemorrhage, as evidenced by HE staining. It has been shown that the reduced neuroprotective effect of $\alpha$-Syn in the spinal cord is at least partly due to inhibition of BCSB destruction and haemorrhage after SCI. Interestingly, there is increasing evidence that $\alpha$-Syn is produced not only in the CNS but also in the periphery. Several studies have shown that there are multiple pathways of $\alpha$-Syn transmission from the periphery to the brain, and 


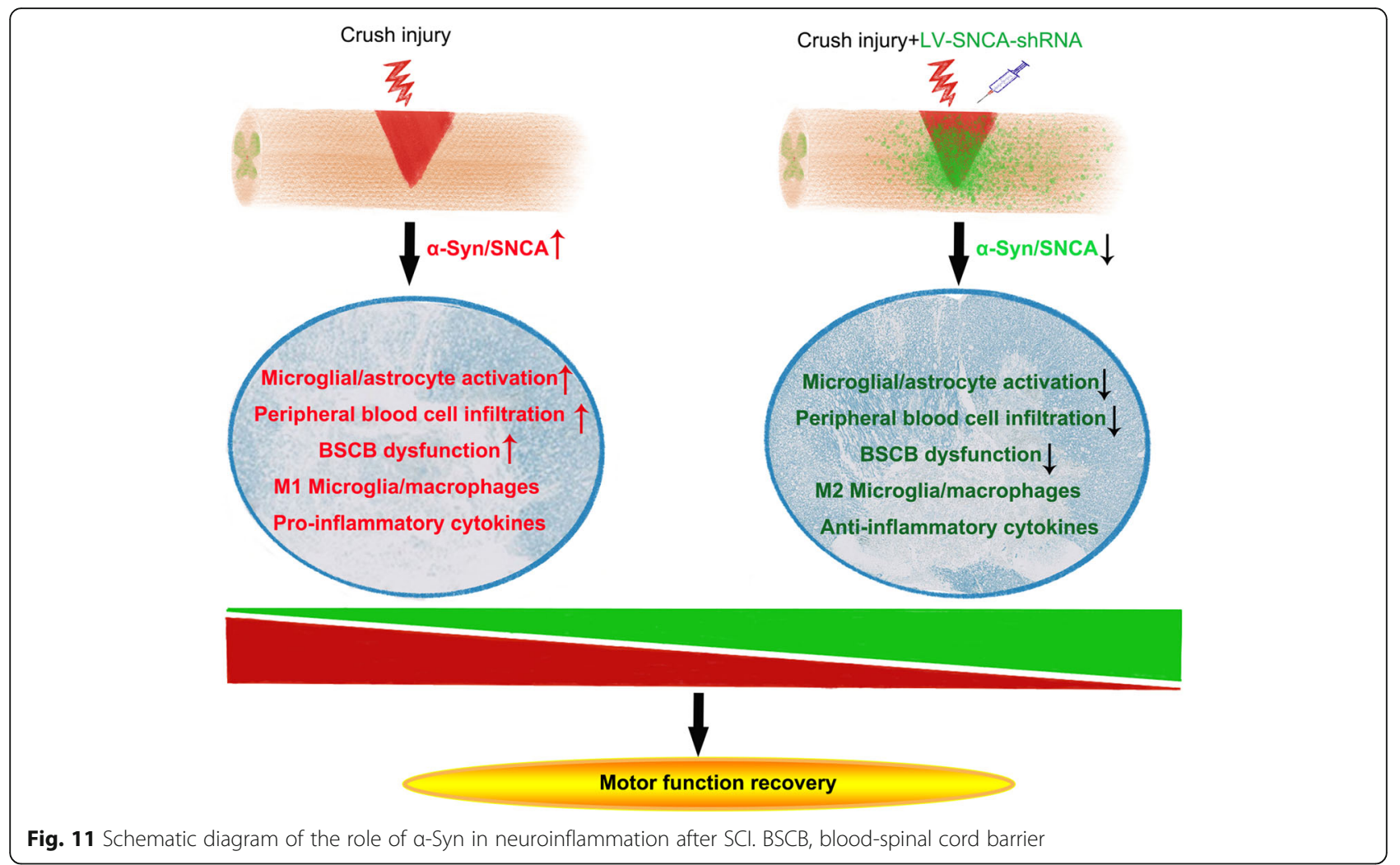

$\alpha$-Syn levels in the plasma are approximately 10 times higher than those in the cerebrospinal fluid (CSF) [52, 53]. Typically, $\alpha$-Syn transport in the blood is controlled by the blood-brain barrier (a dynamic barrier between the peripheral circulatory system and the CNS). Free $\alpha$ Syn protein is transported bidirectionally (from the blood to the brain and in the reverse direction) through this barrier [54]. Based on our data and those from others, we hypothesize that after SCI, reducing $\alpha$-Syn at the SCI site reduces neuroinflammation, ameliorates BCSB dysfunction, and prevents excessive free $\alpha$-Syn in the blood from being transported to the CNS, further improving the homeostatic environment after SCI.

Although LV-SNCA-shRNA was injected early, the in vivo transfection took 4-5 days, so we were unable to observe neuroinflammatory changes in the acute phase in the LV-SNCA-shRNA treatment group. After $\mathrm{SCI}$, considerable infiltration of peripheral monocytes occurred due to the destruction of the BCSB, and we could not determine the impact of these processes on inflammation. Specific cell markers should be detected in subsequent studies. We demonstrated that LVSNCA-shRNA treatment improves neuroinflammation and promotes functional recovery after SCI by promoting the M1/M2 phenotype. However, the exact signalling pathway is not described here, and the relationship between inflammation and synucleinopathies is unclear.
In summary, the data reported here demonstrate that the balance between pro-inflammatory and antiinflammatory microglial/macrophage phenotypes affects the overall inflammatory state following SCI and is also the main factor controlling the survival of neurons.

\section{Conclusion}

Lentivirus-mediated downregulation of $\alpha$-synuclein reduces neuroinflammation and improves functional recovery in rats with SCI. We tested $\alpha$-Syn activation and microglial phenotypes, and we demonstrated that reducing $\alpha$-Syn levels promotes the conversion of microglia from a pro-inflammatory phenotype to an antiinflammatory phenotype and prevents neuronal loss after SCI. Furthermore, we demonstrated that reducing $\alpha$-Syn can improve BCSB function and astrocyte activation after $\mathrm{SCI}$, reduce the occurrence and development of neuroinflammation, and confer a neuroprotective immune microenvironment. This study provides a basis for future research on immune regulation after $\mathrm{SCI}$ with regard to $\alpha$-synucleinopathy.

\section{Supplementary information}

Supplementary information accompanies this paper at https://doi.org/10. 1186/s12974-019-1658-2.

Additional file 1. Lentiviral-SNCA-shRNA transfection efficiency and validation (in vitro experiments) 
Additional file 2:. Expression of glial cells at the injury site on the first day after SCI. (A-B) Representative images showing Iba1+ staining after $\mathrm{SCl}$ by IHC. Scale bars $=250 \mu \mathrm{m}, 100 \mu \mathrm{m}$. (C-D) Representative images showing GFAP+ staining after SCl by IHC. Scale bars $=250 \mu \mathrm{m}, 100 \mu \mathrm{m}$. (E) Quantitative analysis of the mean IOD of Iba1 in panel $A, n=5$. (F) Quantitative analysis of the mean IOD of GFAP in panel $C, n=5$. All data are presented as the mean \pm SD. One-way ANOVA and Tukey's multiple comparisons test were used to analyse differences among groups

\section{Abbreviations}

Arg1: Arginase-1; BBB: Basso-Beattie-Bresnahan; BCSB: Blood-cerebrospinal barrier; CNS: Central nervous system; CNTF: Ciliary neurotrophic factor; CSF: Cerebrospinal fluid; ELISA: Enzyme-linked immunosorbent assay; GCRP: G-coupled receptor protein kinase; GFAP: Glial fibrillary acid protein; GM: Grey matter; HE: Haematoxylin-eosin; Iba1: Ionized calcium-binding adaptor molecule 1; IFN: Interferon; IL: Interleukin; iNOS: Inducible nitric oxide synthase; IOD: Integrated optical density; KD: Knockdown; LFB: Luxol fast blue; LV: Lentivirus; MAPK: Mitogen-activated protein kinase; MMP: Matrix metalloproteinase; NF-kB: Nuclear factor kappa B; RT-qPCR: Quantitative realtime polymerase chain reaction; SCl: Spinal cord injury; shRNA: Short hairpin RNA; TGF: Transforming growth factor; TNF: Tumour necrosis factor; WM: White matter; a-Syn: a-Synuclein

\section{Acknowledgements}

We are grateful for the technical support provided by Peking University Third Hospital Central Laboratory.

\section{Authors' contributions}

MWZ and $\mathrm{HZ}$ conceived and designed the experiments. All the authors participated in performing the experiments and analysing the data. $\mathrm{HZ}$, $M W Z$, and LN wrote, revised, and revised the article. All authors read, revised, and approved the final manuscript.

\section{Funding}

This study was supported by the National Natural Science Foundation of China (No. 1150020073 and 11472018).

\section{Availability of data and materials}

The datasets used and/or analysed during the current study are available from the corresponding author on reasonable request.

\section{Ethics approval and consent to participate}

All experimental procedures were carried out in accordance with the Chinese Guidelines of Animal Care and Welfare, and the study was approved by the Animal Welfare Ethics Branch of the Peking University Bioethics Committee.

\section{Consent for publication}

Not applicable

\section{Competing interests}

The authors declare that they have no competing interests.

Received: 16 May 2019 Accepted: 26 November 2019

Published online: 30 December 2019

\section{References}

1. Ramer LM, Ramer MS, Bradbury EJ. Restoring function after spinal cord injury: towards clinical translation of experimental strategies. Lancet Neurol. 2014;13:1241-56

2. Hayta E, Elden H. Acute spinal cord injury: a review of pathophysiology and potential of non-steroidal anti-inflammatory drugs for pharmacological intervention. J Chem Neuroanat. 2018:87:25-31.

3. Ren H, Chen X, Tian M, Zhou J, Ouyang H, Zhang Z. Regulation of inflammatory cytokines for spinal cord injury repair through local delivery of therapeutic agents. Adv Sci (Weinh). 2018;5:1800529.

4. Sauer RS, Kirchner J, Yang S, Hu L, Leinders M, Sommer C, Brack A, Rittner $\mathrm{HL}$. Blood-spinal cord barrier breakdown and pericyte deficiency in peripheral neuropathy. Ann N Y Acad Sci. 2017;1405:71-88.
5. Kwon BK, Tetzlaff W, Grauer JN, Beiner J, Vaccaro AR. Pathophysiology and pharmacologic treatment of acute spinal cord injury. Spine J. 2004:4:451-64.

6. Mills CD, Kincaid K, Alt JM, Heilman MJ, Hill AM. M-1/M-2 Macrophages and the Th1/Th2 Paradigm. J Immunol. 2000;164:6166-73.

7. Li J, Jia Z, Xu W, Guo W, Zhang M, Bi J, Cao Y, Fan Z, Li G. TGN-020 alleviates edema and inhibits astrocyte activation and glial scar formation after spinal cord compression injury in rats. Life Sci. 2019;222:148-57.

8. Minakaki G, Menges S, Kittel A, Emmanouilidou E, Schaeffner I, Barkovits K, Bergmann A, Rockenstein E, et al. Autophagy inhibition promotes SNCA/ alpha-synuclein release and transfer via extracellular vesicles with a hybrid autophagosome-exosome-like phenotype. Autophagy. 2018;14:98-119.

9. Feng GY, Liu J, Wang YC, Wang ZY, Hu Y, Xia QJ, Xu Y, Shang FF, et al. Effects of alpha-synuclein on primary spinal cord neurons associated with apoptosis and CNTF expression. Cell Mol Neurobiol. 2017;37:817-29.

10. Samuel F, Flavin WP, labal S, Pacelli C, Sri Renganathan SD, Trudeau LE, Campbell EM, Fraser PE, Tandon A. Effects of serine 129 phosphorylation on alpha-synuclein aggregation, membrane association, and internalization. J Biol Chem. 2016;291:4374-85

11. Fogerson SM, van Brummen AJ, Busch DJ, Allen SR, Roychaudhuri R, Banks SM, Klärner FG, Schrader T, et al. Reducing synuclein accumulation improves neuronal survival after spinal cord injury. Exp Neurol. 2016;278:105-15.

12. Qiao H, He X, Zhang Q, Yuan H, Wang D, Li L, Hui Y, Wu Z, Li W, Zhang N. Alpha-synuclein induces microglial migration via PKM2-dependent glycolysis. Int J Biol Macromol. 2019;129:601-7.

13. Basso DM, Beattie MS, Bresnahan JC. A sensitive and reliable locomotor rating scale for open field testing in rats. J Neurotrauma. 1995;12:1-21.

14. Rivlin $\mathrm{AS}$, Tator $\mathrm{CH}$. Objective clinical assessment of motor function after experimental spinal cord injury in the rat. J Neurosurg. 1977;47:577-81.

15. Gong ZMM, Tang ZY, Sun XL. miR-411 suppresses acute spinal cord injury via downregulation of Fas ligand in rats. Biochem Biophys Res Commun. 2018; 501:501-6.

16. Phillips AA, Matin N, Jia M, Squair JW, Monga A, Zheng MMZ, Sachdeva R, et al. Transient hypertension after spinal cord injury leads to cerebrovascular endothelial dysfunction and fibrosis. J Neurotrauma. 2018;35:573-81.

17. Rust $R$, Kaiser $J$. Insights into the dual role of inflammation after spinal cord injury. J Neurosci. 2017:37:4658-60.

18. Blaszczyk L, Maître M, Lesté-Lasserre T, Clark S, Cota D, SHR O, Fénelon VS. Sequential alteration of microglia and astrocytes in the rat thalamus following spinal nerve ligation. J Neuroinflammation. 2018;15:349.

19. Ma Y, Wang J, Wang Y, Yang GY. The biphasic function of microglia in ischemic stroke. Prog Neurobiol. 2017;157:247-72.

20. Beier EE, Neal M, Alam G, Edler M, Wu $\sqcup$, Richardson JR. Alternative microglial activation is associated with cessation of progressive dopamine neuron loss in mice systemically administered lipopolysaccharide. Neurobiol Dis. 2017;108:115-27.

21. Freeman MR. Specification and morphogenesis of astrocytes. Science. 2010; 330:774-8.

22. Rohani MG, Parks WC. Matrix remodeling by MMPs during wound repair. Matrix Biol. 2015;44-46:113-21.

23. Takarada-lemata M, Yoshikawa A, Ta HM, Okitani N, Nishiuchi T, Aida Y, Kamide T, Hattori T, et al. N-myc downstream-regulated gene 2 protects blood-brain barrier integrity following cerebral ischemia. Glia. 2018;66:1432-46.

24. Zhang D, Tang Q, Zheng G, Wang C, Zhou Y, Wu Y, Xuan J, Tian N, et al. Metformin ameliorates BSCB disruption by inhibiting neutrophil infiltration and MMP-9 expression but not direct TJ proteins expression regulation. J Cell Mol Med. 2017;21:3322-36.

25. Squair JW, Ruiz I, Phillips AA, Zheng MMZ, Sarafis ZK, Sachdeva R, Gopaul R, Liu J, et al. Minocycline reduces the severity of autonomic dysreflexia after experimental spinal cord injury. J Neurotrauma. 2018;35:2861-71.

26. Hentrich T, Wassouf Z, Riess O, Julia M. SNCA overexpression disturbs hippocampal gene expression trajectories in midlife. Aging. 2018:10:4024-41.

27. Chiba-Falek O. Structural variants in SNCA gene and the implication to synucleinopathies. Curr Opin Genet Dev. 2017;44:110-6.

28. Poehler AM, Xiang W, Spitzer P, May VE, Meixner H, Rockenstein E, Chutna $\mathrm{O}$, et al. Autophagy modulates SNCA/alpha-synuclein release, thereby generating a hostile microenvironment. Autophagy. 2014;10:2171-92.

29. Koprich JB, Kalia LV, Brotchie JM. Animal models of alpha-synucleinopathy for Parkinson disease drug development. Nat Rev Neurosci. 2017:18:515-29.

30. Bachhuber T, Katzmarski N, JF MC, Loreth D, Tahirovic S, Kamp F, AbouAjram C, Nuscher B, et al. Inhibition of amyloid-beta plaque formation by alpha-synuclein. Nat Med. 2015;21:802-7. 
31. Sakurai M, Kawamura T, Nishimura H, Suzuki H, Tezuka F, Abe K. Induction of Parkinson disease-related proteins in motor neurons after transient spinal cord ischemia in rabbits. J Cereb Blood Flow Metab. 2009;29:752-8.

32. Busch DJ, Morgan JR. Synuclein accumulation is associated with cell-specific neuronal death after spinal cord injury. J Comp Neurol. 2012;520:1751-71.

33. Kim T, Mehta SL, Kaimal B, Lyons K, Dempsey RJ, Vemuganti R. Poststroke induction of alpha-synuclein mediates ischemic brain damage. J Neurosci. 2016;36:7055-65.

34. Kim T, Mehta SL, Morris-Blanco KC, Chokkalla AK, Chelluboina B, Lopez M, Sullivan $\mathrm{R}$, et al. The microRNA miR-7a-5p ameliorates ischemic brain damage by repressing a-synuclein. Sci Signal. 2018;11:560.

35. Liu J, Wang X, Lu Y, Duan C, Gao G, Lu L, Yang H. Pink1 interacts with alpha-synuclein and abrogates alpha-synuclein-induced neurotoxicity by activating autophagy. Cell Death Dis. 2017:8:e3056

36. Yan DY, Liu C, Tan X, Ma Z, Wang C, Deng Y, Liu W, Xu ZF, Xu B. MnInduced neurocytes injury and autophagy dysfunction in alpha-synuclein wild-type and knock-out mice: highlighting the role of alpha-synuclein. Neurotox Res. 2019;36(1):66-80

37. Martínez-Morillo E, García Hernández P, Begcevic I, Kosanam H, Prieto García B, Alvarez Menéndez FV, Diamandis EP. Identification of novel biomarkers of brain damage in patients with hemorrhagic stroke by integrating bioinformatics and mass spectrometry-based proteomics. J Proteome Res. 2014;13:969-81.

38. Uryu K, Chen XH, Martinez D, Browne KD, Johnson VE, Graham DI, Lee VM, et al. Multiple proteins implicated in neurodegenerative diseases accumulate in axons after brain trauma in humans. Exp Neurol. 2007;208: 185-92.

39. Salem SA, Allsop D, Mann DM, Tokuda T, El-Agnaf OM. An investigation into the lipid-binding properties of alpha-, beta- and gamma-synucleins in human brain and cerebrospinal fluid. Brain Res. 2007;1170:103-11.

40. Kenney K, lacono D, Edlow BL, Katz DI, Diaz-Arrastia R, Dams-O'Connor K, Daneshvar DH, Stevens A, et al. Dementia after moderate-severe traumatic brain injury: coexistence of multiple proteinopathies. J Neuropathol Exp Neurol. 2018;77:50-63.

41. Yeh TS, Ho YC, Hsu CL, Pan SL. Spinal cord injury and Alzheimer's disease risk: a population-based, retrospective cohort study. Spinal Cord. 2018;56: 151-7.

42. Yeh TS, Huang YP, Wang HI, Pan SL. Spinal cord injury and Parkinson's disease: a population-based, propensity score-matched, longitudinal followup study. Spinal Cord. 2016:54:1215-9.

43. Voet S, Srinivasan S, Lamkanfi M, van Loo G. Inflammasomes in neuroinflammatory and neurodegenerative diseases. EMBO Mol Med. 2019.

44. Qiao H, He X, Zhang Q, Zhang N, Li L, Hui Y, Li W, Wang D, Wu Z. Alphasynuclein induces microglial cell migration through stimulating HIF-1alpha accumulation. J Neurosci Res. 2017:95:1809-17.

45. Milich LM, Ryan CB, Lee JK. The origin, fate, and contribution of macrophages to spinal cord injury pathology. Acta Neuropathol. 2019;137: 785-97

46. Rodriguez-Perez Al, Sucunza D, Pedrosa MA, Garrido-Gil P, Kulisevsky J, Lanciego JL, Labandeira-Garcia JL. Angiotensin type 1 receptor antagonists protect against alpha-synuclein-induced neuroinflammation and dopaminergic neuron death. Neurotherapeutics. 2018;15:1063-81.

47. Harms AS, Delic V, Thome AD, Bryant N, Liu Z, Chandra S, Jurkuvenaite A, West AB. alpha-Synuclein fibrils recruit peripheral immune cells in the rat brain prior to neurodegeneration. Acta Neuropathol Commun. 2017;5:85.

48. Qiao H, Zhang Q, Yuan H, Li Y, Wang D, Wang R, He X. Elevated neuronal alpha-synuclein promotes microglia activation after spinal cord ischemic/ reperfused injury. Neuroreport. 2015;26:656-61.

49. Gu Y, Cheng $X$, Huang $X$, Yuan $Y$, Qin S, Tan Z, Wang D, et al. Conditional ablation of reactive astrocytes to dissect their roles in spinal cord injury and repair. Brain Behav Immun. 2019:80:394-405

50. Hsu JY, Bourguignon LY, Adams CM, Peyrollier K, Zhang H, Fandel T, Cun CL, Werb Z, Noble-Haeusslein LJ. Matrix metalloproteinase-9 facilitates glial scar formation in the injured spinal cord. J Neurosci. 2008;28:13467-77.

51. Noble LJ, Donovan F, Igarashi T, Goussev S, Werb Z, et al. Matrix metalloproteinases limit functional recovery after spinal cord injury by modulation of early vascular events. J Neurosci. 2002;22:7526-35.

52. Hong Z, Shi M, Chung KA, Quinn JF, Peskind ER, Galasko D, Jankovic J, Zabetian CP, et al. DJ-1 and alpha-synuclein in human cerebrospinal fluid as biomarkers of Parkinson's disease. Brain. 2010:133:713-26.
53. Shi M, Zabetian CP, Hancock AM, Ginghina C, Hong Z, Yearout D, Chung KA, Quinn JF, et al. Significance and confounders of peripheral DJ-1 and alpha-synuclein in Parkinson's disease. Neurosci Lett. 2010;480:78-82.

54. Matsumoto J, Stewart T, Sheng L, Li N, Bullock K, Song N, Shi M, Banks WA, Zhang J. Transmission of alpha-synuclein-containing erythrocyte-derived extracellular vesicles across the blood-brain barrier via adsorptive mediated transcytosis: another mechanism for initiation and progression of Parkinson's disease? Acta Neuropathol Commun. 2017;5:71.

\section{Publisher's Note}

Springer Nature remains neutral with regard to jurisdictional claims in published maps and institutional affiliations.
Ready to submit your research? Choose BMC and benefit from:

- fast, convenient online submission

- thorough peer review by experienced researchers in your field

- rapid publication on acceptance

- support for research data, including large and complex data types

- gold Open Access which fosters wider collaboration and increased citations

- maximum visibility for your research: over $100 \mathrm{M}$ website views per year

At BMC, research is always in progress.

Learn more biomedcentral.com/submissions 